\title{
What do randomized controlled trials say about virtual rehabilitation in stroke? A systematic literature review and meta- analysis of upper-limb and cognitive outcomes
}

Anna Aminov ${ }^{1}$ (D), Jeffrey M. Rogers ${ }^{2}$, Sandy Middleton ${ }^{1}$, Karen Caeyenberghs ${ }^{3,4}$ and Peter H. Wilson ${ }^{1,3,4^{*}}$

\begin{abstract}
Background: Virtual-reality based rehabilitation (VR) shows potential as an engaging and effective way to improve upper-limb function and cognitive abilities following a stroke. However, an updated synthesis of the literature is needed to capture growth in recent research and address gaps in our understanding of factors that may optimize training parameters and treatment effects.

Methods: Published randomized controlled trials comparing VR to conventional therapy were retrieved from seven electronic databases. Treatment effects (Hedge's g) were estimated using a random effects model, with motor and functional outcomes between different protocols compared at the Body Structure/Function, Activity, and Participation levels of the International Classification of Functioning.

Results: Thirty-three studies were identified, including 971 participants (492 VR participants). VR produced small to medium overall effects ( $g=0.46 ; 95 \% \mathrm{Cl}: 0.33-0.59, p<0.01$ ), above and beyond conventional therapies. Small to medium effects were observed on Body Structure/Function ( $q=0.41 ; 95 \% \mathrm{Cl}: 0.28-0.55 ; p<0.01)$ and Activity outcomes ( $g=0.47 ; 95 \% \mathrm{Cl}: 0.34-0.60, p<0.01)$, while Participation outcomes failed to reach significance $(g=0.38$; 95\% Cl: $-0.29-1.04, p=0.27)$. Superior benefits for Body Structure/Function $(g=0.56)$ and Activity outcomes $(g=0.62)$ were observed when examining outcomes only from purpose-designed VR systems. Preliminary results $(k=4)$ suggested small to medium effects for cognitive outcomes ( $g=0.41 ; 95 \% \mathrm{Cl}: 0.28-0.55 ; p<0.01$ ). Moderator analysis found no advantage for higher doses of VR, massed practice training schedules, or greater time since injury.

Conclusion: VR can effect significant gains on Body Structure/Function and Activity level outcomes, including improvements in cognitive function, for individuals who have sustained a stroke. The evidence supports the use of VR as an adjunct for stroke rehabilitation, with effectiveness evident for a variety of platforms, training parameters, and stages of recovery.
\end{abstract}

Keywords: Cognition, Meta-analysis, Motor performance, Rehabilitation, Stroke, Virtual reality

* Correspondence: peterh.wilson@acu.edu.au

${ }^{1}$ School of Psychology, Faculty of Health Sciences, Australian Catholic

University, Sydney, NSW, Australia

${ }^{3}$ School of Psychology, Australian Catholic University, Melbourne, VIC,

Australia

Full list of author information is available at the end of the article 


\section{Background}

Stroke is one of the leading global causes of disability $[1,2]$, with over 17 million individuals worldwide sustaining a stroke each year [2]. Although stroke mortality is decreasing with improvements in medical technology [3], the neurological trauma resulting from stroke can be devastating, and the majority of stroke survivors have substantial motor $[4,5]$, cognitive [6-9] and functional rehabilitation needs $[3,10,11]$, and much reduced quality of life $[3,12,13]$. Targeted rehabilitation can help address some of these post-stroke deficits, however, historically, many individuals, in particular patients with cognitive impairment, have difficulty engaging in standard therapies [14-16] at a level that will produce meaningful and lasting improvements [16-19]. Enriched and interactive rehabilitation programs are clearly needed to minimize functional disability $[13,20]$, increase participation in age-appropriate roles and activities [21], lead to greater motivation and treatment compliance [17, 22], and reduce the long-term expense of care in stroke survivors $[20,23,24]$.

\section{Virtual reality}

Virtual reality refers to simulated interactions with environments and events that are presented to the performer with the aid of technology. These so-called virtual environments may mirror aspects of the real world or represent spaces that are far removed from it, while allowing various forms of user interaction through movement and/or speech [25]. Virtual reality based rehabilitation, or Virtual Rehabilitation (VR), shows considerable promise as a safe, engaging, interactive, patient-centered and relatively inexpensive medium for rehabilitation training [26-31]. VR has the potential to target a wide range of motor, functional, and cognitive issues [23], affords methods that automatically record and track patient performance [32], and offers a high level of flexibility and control over therapeutic tasks $[17,18,33]$. This scalability allows patients to train at the highest intensity that would be possible for their individual ability [34], while keeping the experience of interaction with therapeutic tasks enjoyable and compelling $[17,29]$. At the same time, VR may enable patients with a neurodisability (like stroke) to practice without excessive physical fatigue $[32,35]$ which otherwise may deter continued effort and engagement in therapy [36, 37].

Currently, there are two main types of VR: purposedesigned Virtual Environments (VE) and Commercial Gaming (CG) systems. Both types of systems can provide augmented feedback, additional forms of sensory feedback about the patient's movement over and above the feedback that is provided as a natural consequence of the movement itself $[11,38]$. VE systems are often designed by rehabilitation scientists (and others) to enhance the delivery of augmented feedback in order to develop the patient's sense of position in space [39-41], to reinforce different movement parameters (like trajectory and endpoint) and reduce extraneous movements (e.g. excessive trunk displacement) [42, 43].

VE systems are also more likely to involve specially designed tangible user interfaces used in mixed reality rehabilitation systems [13] or training of daily functional activities [44]. By comparison, CG rehabilitation systems are typically "off-the-shelf" devices such as Wii (Nintendo), Xbox (Microsoft) and PlayStation (Sony), which have the advantage of being readily available and relatively inexpensive when compared with VE systems [11]. On the other hand, CG systems are typically designed for able-bodied participants and may not consider the physiological, motor, and cognitive aspects of recovery in rehabilitation, and may lack the scalability of purpose-designed VE systems [45].

\section{Systematic reviews comparing VE and CG systems}

There is conflicting evidence about the relative effectiveness of VE- and CG-based VR systems. In a recent Cochrane review of VR following stroke [46], VE systems demonstrated a significant treatment effect on upper-limb function when compared to controls $(d=0.42 ; 95 \% \mathrm{CI}$ : 0.07-0.76), while the effect for CG systems failed to reach significance $(d=0.50 ; 95 \% \mathrm{CI}$ : $-0.04-1.04)$; a caveat, however, was that only two of nine studies $(22 \%)$ in these comparisons were CG-based. In contrast, a meta-analysis by Lohse and colleagues of VR following stroke [11] found no significant difference between VE $(g=0.43$, based on 13 studies) and CG interventions ( $g=0.76$, based on three studies) on Body Structure/Function level outcomes. For Activity level outcomes, CG interventions showed a large but non-significant effect $(g=0.76, p=0.14)$, but was based on only four of 26 studies (15\%); VE interventions, however, showed a significant treatment effect $(g=0.54, p$ $<.001)$. Taken together, these two reviews suggest benefits of VE systems, while previous analyses of CG treatment effects have been underpowered and inconclusive.

\section{Cognition and VR}

Cognitive impairments, including difficulties in attention, language, visuospatial skills, memory, and executive function are common and persistent sequelae of stroke [14, 47] and exert considerable influence on rehabilitation outcomes [48]. Cognitive dysfunction may reduce the ability to (re-)acquire motor [25, 49-52] and functional skills [47], and decrease engagement and participation in rehabilitation program $[48,53]$. While the important role of cognition in both conventional and VR-based rehabilitation is increasingly recognized 
[52-54] the impact of VR on cognitive function has not yet been formally evaluated in a quantitative review.

\section{Analysis of individual domains of functioning}

The World Health Organization's International Classification of Functioning, Disability, and Health (ICF-WHO [55]) is currently one of the most widely used classification systems. It is a foundation for understanding outcome effects in clinical practice [56] and the preferred means for translating clinical findings in a patient-centered manner [56]. Under the ICF-WHO, disability and functioning are seen to arise by the interaction of the health condition, the environment, and personal factors, and can be measured at three main levels: (i) Body Structure/Function, (ii) Activity (or skill), and (iii) Participation. The ICF-WHO has been used to classify outcome measures in studies of VR (for example [57]) and in recent systematic reviews [11, 58, 59]. A brief critique of these reviews reveals a number of important conclusions, but also some significant gaps in the research.

An early systematic review by Crosbie and colleagues [60] examined the efficacy of VR for stroke upon motor and cognitive outcomes. Of the 11 studies reviewed (up to 2005), only five addressed upper-limb function and two addressed cognitive outcomes. Overall, the review reported significant benefits of VR, but only three studies were RCTs and no effect size estimates were reported. At around the same time, a systematic review by Henderson and colleagues [61] showed that there was very good evidence that immersive VR was more beneficial than no therapy for upper-limb rehabilitation in adult stroke, but insufficient evidence for non-immersive VR. Comparisons with traditional physical therapy were less impressive, however.

A 2016 systematic review by Vinas-Diz and colleagues [62] included both controlled clinical trials and randomized controlled trials (RCTs) in stroke, and spanned 2009-2014. The review included 25 papers: four systematic reviews $[19,46,63,64]$ and 21 original trials. Evidence for treatment efficacy on upper-limb function was strong on a mix of measures like the Fugl-Meyer Test, Wolf Motor Function Test, and Motricity Index. However, a quantitative analysis of the effects was not undertaken, and important aspects of treatment implementation like dose and session scheduling were not formally examined.

A recent systematic review by Santos-Palma and colleagues [58] examined the efficacy of VR on motor outcomes for stroke using the ICF-WHO framework, covering work published up to June 2015. Of the studies deemed high quality, 20 examined outcomes at the Body Structure/Function level, 17 at the Activity level, and eight examined Participation. Intriguingly, positive outcomes were evident only at the Body Structure/Function level, while results for Activity and Participation were not conclusive. Unfortunately, only three studies addressed manual ability at the Activity level, which severely limited any evaluation of skill-specific effects.

In a combined systematic review and meta-analysis of 37 RCTs published between 2004 and 2013, Laver and colleagues [46] present a more comprehensive examination of the effects of VR on upper-limb function. As well, they classified outcomes broadly into upper-limb function, Activities of Daily Living (ADLs) and other aspects of motor function. In general, study quality was low, and the risk of bias high, in roughly one-half of the studies. Outcomes were significant for upper-limb function $(d=0.28)$ and ADLs $(d=0.43)$, but somewhat smaller than those reported by Lohse and colleagues [11]. Results for other aspects of motor function, including several at what may be considered the Body Structure/Function level, were non-significant. Dose varied considerably between studies, ranging from less than $5 \mathrm{~h}$ to more than $21 \mathrm{~h}$ in total. In general, studies that used higher doses ( $>15 \mathrm{~h}$ of therapy) were reported as more effective. Unfortunately, results could not be pooled for cognitive outcomes, and the importance of additional treatment implementation parameters like training frequency and duration, and the impact of specific study design factors including the recovery stage of participants and type of control group (i.e. active vs passive) were not determined.

An updated systematic review by Laver and colleagues [65], included an additional 35 studies that reported outcomes for upper limb function and activity. A subset of only 22 studies that compared VR with conventional therapy showed no significant effect of VR on upperlimb function $(d=0.07)$. As well, there was no significant difference between higher ( $>15 \mathrm{~h}$ of therapy), and lower levels of dose. However, when VR was used in addition to usual care (10 studies; 210 participants), there was a significant effect on upper-limb outcomes $(d=0.49)$. As before, no significant difference was shown between high and low dose studies. Unfortunately, analysis of cognitive outcomes, and moderator analyses including study quality, and implementation parameters (e.g., daily intensity, weekly intensity, treatment frequency, and total number of sessions) were not included in the updated review. As well, the assessment of study quality was limited to the 5-item GRADE system, the ICF classification system was not given full consideration, and no distinction was drawn between treatment as usual (TAU) and active control groups (TAU + some form of additional therapy).

Taken together, recent reviews on the use of VR for adult stroke show encouraging evidence of efficacy at the level of Body Structure/Function, but mixed results for Activity and ADLs, and a paucity of evidence bearing 
on Participation. The impact and effectiveness of VR on cognitive outcomes also remains poorly understood, despite the important role of cognitive dysfunction in learning and rehabilitation [17, 18], and increased evidence of interconnection between cognitive function and motor deficits at the Body Structure/Function, Activity and Participation levels of the ICF [52]. VEbased platforms have been suggested to be superior to CG approaches [46] in promoting motor function, but until recently there have been few CG studies available for analysis. As well, other design factors that may moderate treatment effects (like stage of recovery, control group type) have either not been explored or are too few in number to draw firm conclusions. There has been considerable variation in the total dose of VR therapy $[46,60]$, and no analysis has yet tested the doseresponse relationship in moderator analyses. Finally, the bulk of conclusions have relied on qualitative synthesis, and there is a paucity of quantitative analysis of empirical data to inform opinion.

In view of limitations in past reviews and continued acceleration in VR the aim of our review was to conduct a systematic literature review and meta-analysis to reevaluate the strength of evidence bearing on VR of upper-limb function and cognition in stroke. This review is critical given evidence that stroke rehabilitation needs to better optimize intervention techniques during the recovery windows that exist in the acute phase [66] and beyond. Focusing only on RCTs, we consider outcomes across levels of the ICF-WHO, and analyze the moderating effect of design factors and dose-related parameters.

\section{Methods}

The current review was conducted and reported in accordance with the Preferred Reporting Items for Systematic Reviews and Meta-Analyses (PRISMA) statement [67], it should be noted that the protocol was not registered.

\section{Data sources and search strategy}

Scopus, Cochrane Database, CINAHL, The Allied and Complementary Medicine Database, Web of Science, MEDLINE, Pre-Medline, PsycEXTRA, and PsycINFO databases were systematically searched from inception until 28 June 2017. Boolean search terms included the following: "stroke, cerebrovascular disease, or cerebrovascular attack" and "Virtual reality, Augment" reality, virtual gam" (see Appendix for an example of the full MEDLINE search strategy).

\section{Inclusion and exclusion criteria}

RCT studies published in English in peer-reviewed journals, utilizing a VR intervention to address either motor (upper-limb), cognitive, or activities of daily living in stroke patients were included in the current review (see Fig. 1). VR was defined as a type of user-computer interface that involves real-time simulation of an activity/environment, enabling the user to interact with the

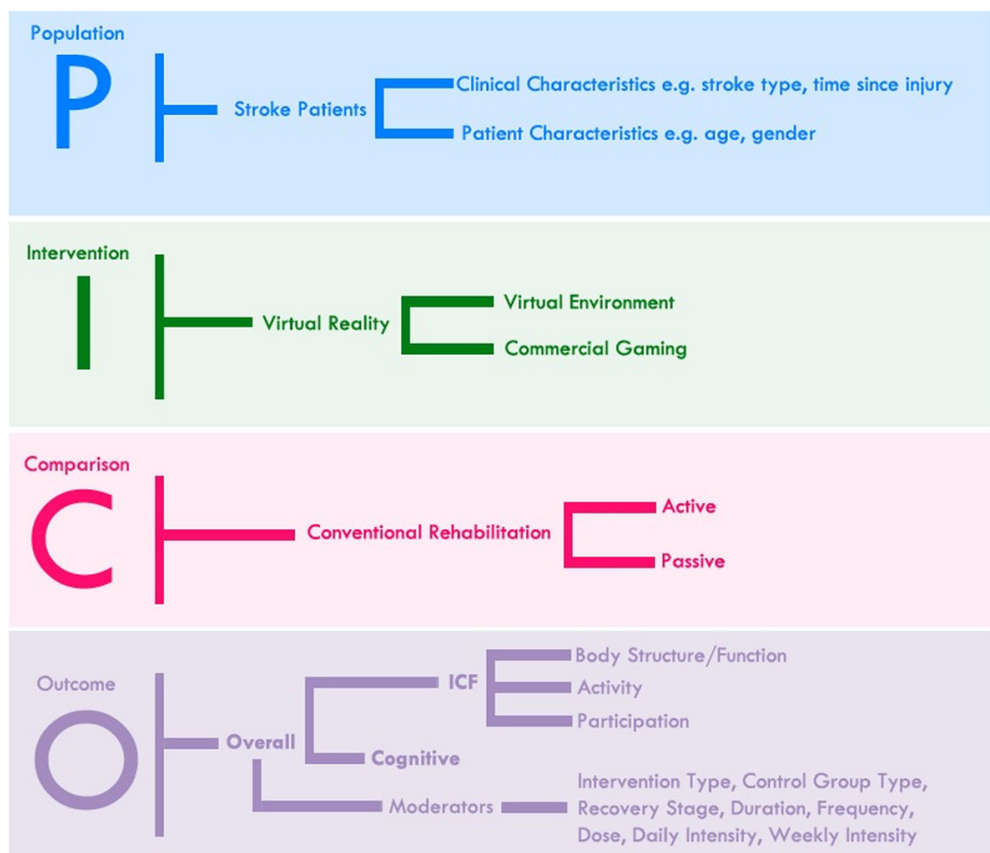

Fig. 1 Population, Intervention, Comparison, Outcome (PICO) Question and the main variables included in the systematic literature review and meta-analysis 
environment using motor actions and sensory systems. Comparison groups included "usual care", "standard care" or "conventional therapy", involving physical therapy and/or occupational therapy. Studies were excluded that applied a "hybrid" approach combining virtual reality with exogenous stimulation or robotics, targeted lower limb function, recruited a mixed study cohort including non-stroke participants, or did not utilize motor, cognitive, or participation outcome measures.

\section{Identification of relevant studies and data extraction}

The eligibility assessment was performed independently using a standardized protocol by two of the authors (AA and JR). After deleting duplicate papers, the title and abstract of all articles were screened by the authors to assess suitability for inclusion. Those considered potentially eligible were read in full. In addition, reference lists of relevant reviews were searched by hand. The last hand search was performed 28 June 2017. For articles meeting inclusion criteria, data on study design, participant characteristics, and intervention outcomes were extracted by two of the authors (AA and JR). Disagreements between reviewers were resolved by consensus.

Extracted VR outcomes were organized according to the three levels of functioning classified by the ICFWHO [55] including: (i) Body Structure/Function, which refers to physiological functions of body systems (e.g. Fugl Meyer Assessment); (ii) Activity, which refers to the execution of tasks or actions (e.g. Box and Blocks Test); and (iii), Participation, which refers to involvement in life situations (e.g. Motor Activity Log [57]).

\section{Quality assessment}

Two authors (AA and PW) assessed the risk of bias of each included article using the Physiotherapy Evidence Database (PEDro) Scale [68]. The PEDro Scale rates methodological quality across 11 bias reducing items relating to the domains of Selection, Performance, Detection, Information, and Attribution biases [69]. Studies with PEDro total scores from 6 to 10 were considered high quality [70]; scores below 6 were considered fair quality. Disagreements between reviewers were resolved by consensus.

\section{Quantitative analysis}

From the published manuscript, post-intervention means and standard deviations on each outcome measure, $p$ values, and sample sizes for the experimental and control groups were entered into Comprehensive Meta-Analysis (CMA; Biostat, Englewood, NJ, USA) version 3.3.070. A random-effects model was used to compute the effect size estimate, Hedge's $g$, a variation of Cohen's $d$ that corrects for small sample sizes. The magnitude of Hedge's $g$ was categorized as follows: small $(\geq 0.2)$, medium $(\geq 0.5)$ and large $(\geq 0.8)$ [71]. Pooled effect sizes were calculated by aggregating the mean effect sizes weighted by each study's sample size, and the 95\% confidence intervals (CI) and $z$ scores based on the overall mean and standard error. Meta-analysis was only performed in cases where there was more than one study in each group [72]. Effect size outcomes favoring VR were assigned a positive value while effects favoring the control condition (i.e. treatment-as-usual) had a negative value. Heterogeneity was formally assessed with the $I^{2}$ statistic, where an $I^{2}$ value greater than $50 \%$ indicated significant heterogeneity [71]. The risk of publication bias was assessed using the Classic failsafe $N$ and Egger's regression test (2-tailed $p$ value). Finally, moderator analyses were conducted using the $Q$ statistic to estimate the likelihood of a given variable moderating observed effect sizes. A total of ten moderator variables were examined, including five design factors, and five implementation parameters (See Table 1).

\section{Results}

Following removal of duplicates, 17,300 records were screened for eligibility. Following the selection process depicted in Fig. 2, a final sample of 31 articles was identified for inclusion in this review. Twenty-eight studies [13, 21, 44, 57, 73-96] utilized an upper-limb training intervention approach, one also aimed to improve cognitive function [97], and two studies [53, 54] targeted cognitive function alone. The pool of studies included work conducted in the UK, Korea, Spain, USA, Brazil, Israel, Sweden, Australia, and New Zealand (see Table 2). Of the 31 articles, two presented two separate studies for analysis [21, 91], providing a total of 33 independent studies. All studies used an RCT design, comparing 492 participants receiving VR (per study $M=14.9, S D=10.9$ ) with 479 participants receiving Conventional Therapy (CT; per study $M=14.5, S D=11.4$ ).

\section{Participant characteristics}

Sample sizes ranged from 4 to 62 participants per group. Eight studies had less than 10 participants in the VR group [21, 57, 76, 79, 81, 83, 84, 90], while only five studies had over 20 participants (range, 20-59) in the VR group [74, 77-79, 86] (see Table 2). The average age was 60.0 years $(S D=6.3$ years, range $48.2-74.1)$. The average time post-stroke for each study (based on 29 independent studies, four studies [54, 90, 91, 97] did not report time post-stroke) varied considerably from 1.9 weeks to 427.8 weeks $(M=79.6$ weeks, $S D=105.2)$. This included seven studies [21, 44, 53, 74, 76, 86, 91] (21\%) conducted during the sub-acute ( $\leq$ three months) 
Table 1 Moderators included in the analyses

\begin{tabular}{ll}
\hline Moderator Type & Definition \\
\hline $\begin{array}{l}\text { Design factors } \\
\text { Intervention Type }\end{array}$ & Virtual Environment (VE) design or Computerized Gaming (CG) system \\
$\begin{array}{l}\text { Simulation Type } \\
\text { Study Quality }\end{array}$ & Modervention targeting hand/finger function or interventions targeting overall upper-limb function \\
Recovery Stage & Sub-Acute ( $\leq 3$ months post-stroke) or Chronic ( $>3$ months post-stroke) \\
Control Group Type & Passive (treatment as usual) or Active (treatment as usual + additional rehabilitation) comparison control group \\
Implementation Parameters & \\
Duration & Total number of sessions per intervention (low: $<15$ sessions; high: $\geq 15$ sessions) \\
Frequency & Number of sessions per week (low: $\leq 3$ sessions; high: $>3$ sessions) \\
Dose & Total number of minutes per intervention (low: $<400$ min; medium: $400-800$ min; high: > 800 min) \\
Daily Intensity & Minutes per session (low: $\leq 30$ min; high: $>30$ min) \\
Weekly Intensity & Total minutes of virtual rehabilitation per week (low: $\leq 100$ min; high: $>100$ min) \\
\hline
\end{tabular}

stage (range $1.9-10.3$ weeks, $M=3.86$ weeks, $S D=3.23$ ), while the remainder completed VR interventions during the chronic (> 3 months post-stroke) stage (range 17.2427.8 weeks, $M=127.40$ weeks, $S D=132.5)$. Seventeen studies [13, 21, 53, 57, 74, 77, 79-82, 85, 87, 91, 92, 94] included both ischemic and hemorrhagic stroke patients, three included only ischemic stroke patients [78, 86, 93],

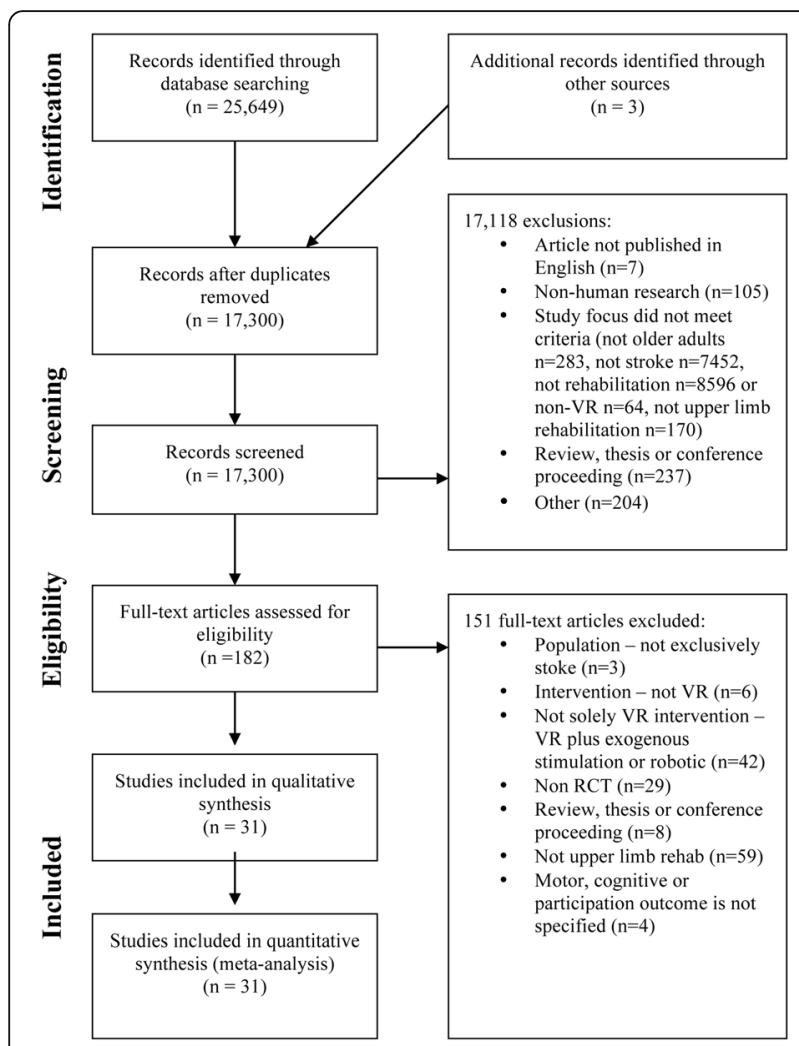

Fig. 2 Four-phase Preferred Reporting Items for Systematic Reviews and Meta-Analyses (PRISMA) flow diagram, showing the process for identifying and screening of the articles for inclusion and exclusion in the systematic literature review and meta-analysis and 11 did not report specific details about stroke type. Only three studies $[21,74]$ reported data on stroke severity, two utilizing the National Institutes of Health Stroke Scale (NIHSS) and one study [86] used the Canadian Neurological Scale of Stroke Severity.

\section{VR and control interventions}

Of the 33 independent studies, 19 used a VE approach and 14 studies evaluated a CG-based therapy (see Table 3). VE interventions involved either video capture or tabletop systems. The former required the patient to be seated in front of a wall display while grasping a sensor, such as the Reinforced Feedback in Virtual Environment system [77-79] and the Rehab Master game-based VE system [75, 76]. Tabletop systems involved multitouch display technologies (e.g. [92, 94]), requiring finger touch response [94] or the manipulation of tangible user interfaces. CG therapies included Wii (Nintendo [73, 74, 80, 83, 86, 91]), Xavix [83], EyeToy (PlayStation [88]), IREX system [53, 82, 97], Xbox Kinect [81, 89], or a combination of systems [95]. All but two intervention programs (93\%) took place in a hospital, one [90] was homebased and another provided rehabilitation at a local community center [92]. Only one study [85] reported on the number of repetitions per session.

All VR and CT group participants received $\mathrm{CT}$. In most of the included studies, this "treatment as usual" was only described in limited terms, but typically involved aspects of either physio- or occupational therapy (see Table 3). In 21 studies, CT group participants also received additional rehabilitation interventions, to match the additional time in therapy provided to participants randomized to VR. These socalled "active" control group interventions included, for example, additional physio- and occupational therapy [83], or additional standard therapy tailored 


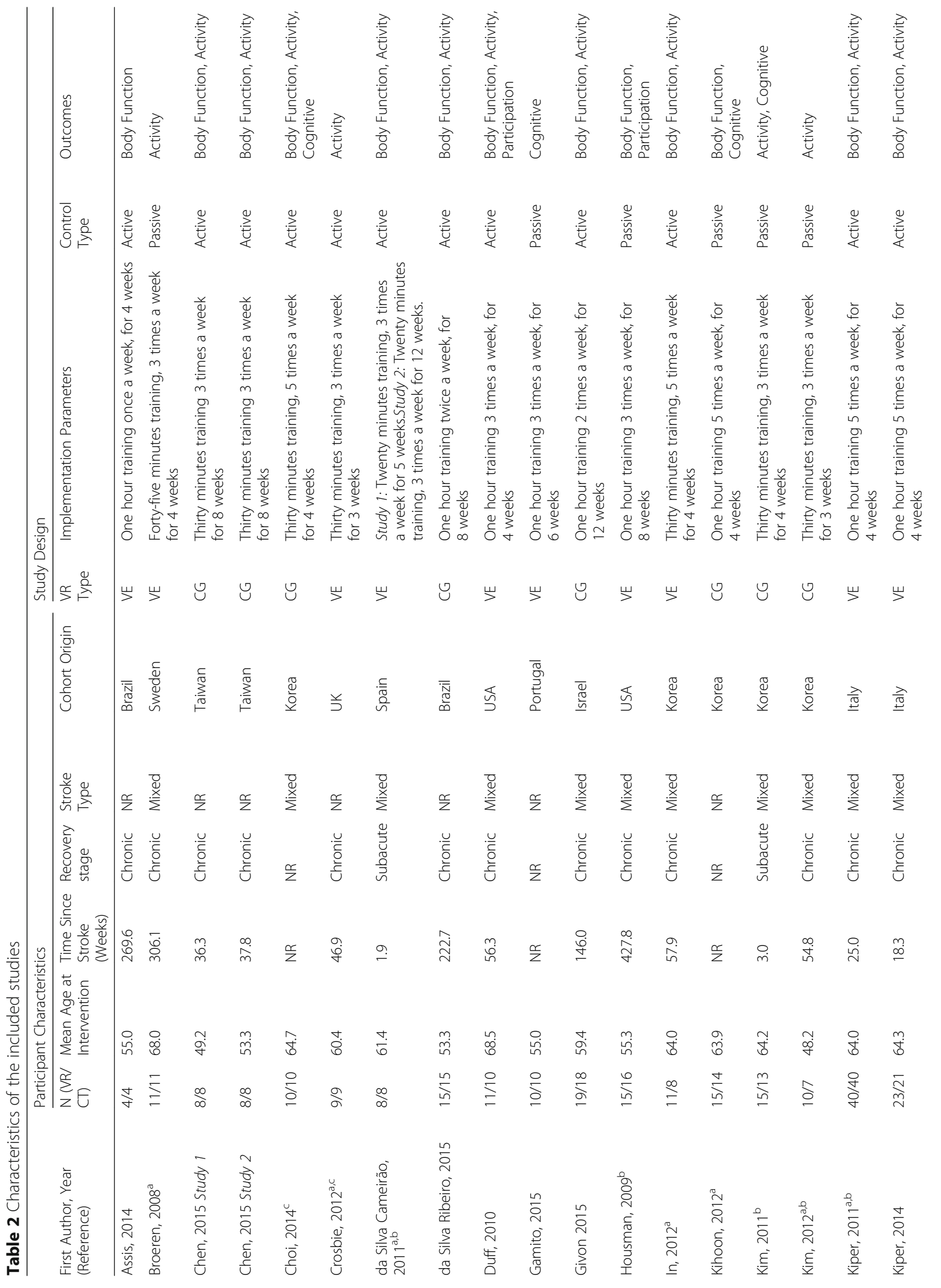




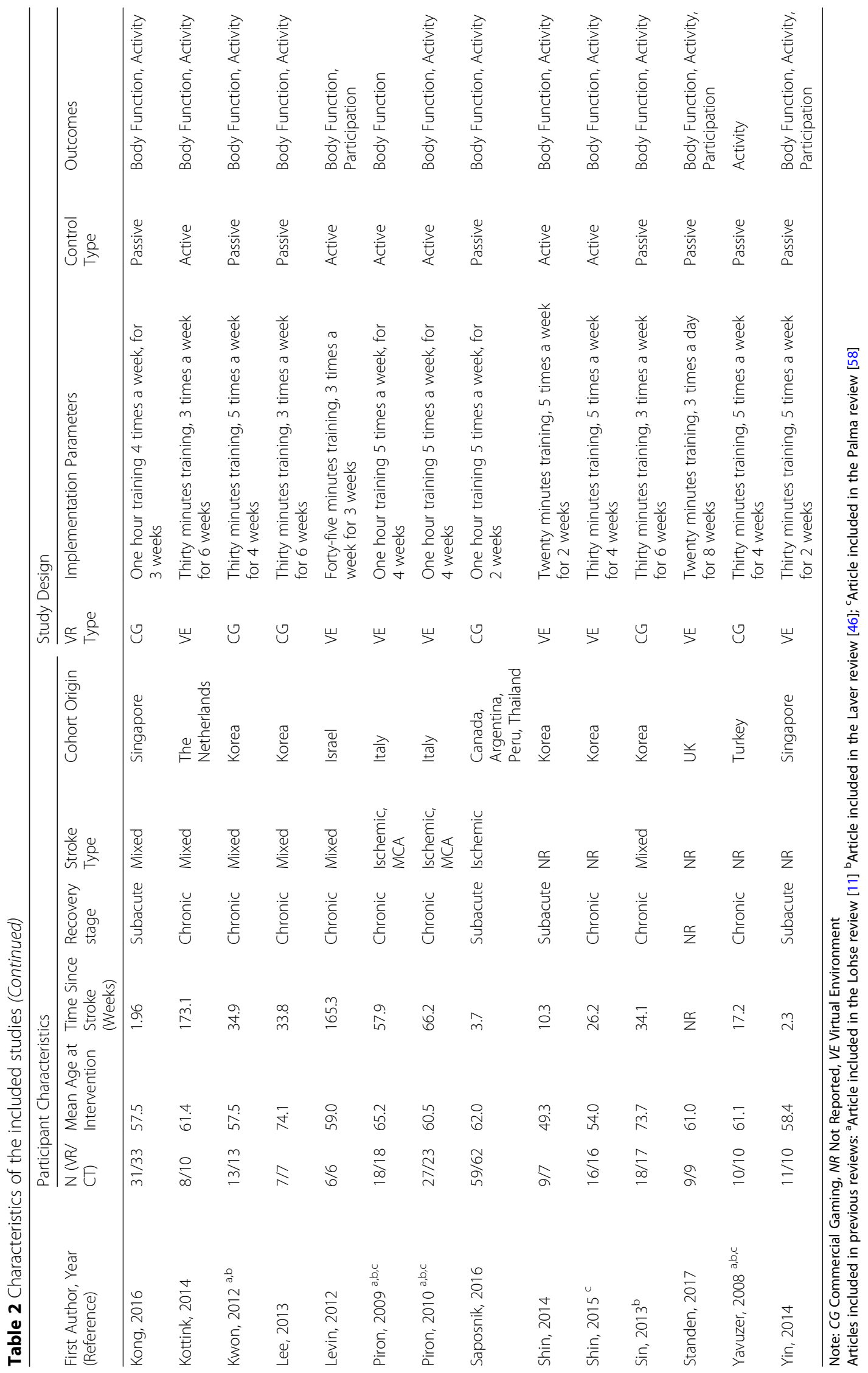


Table 3 Description of the virtual rehabilitation interventions, conventional control group therapies, and additional control treatments, when applicable

\begin{tabular}{|c|c|c|}
\hline $\begin{array}{l}\text { First } \\
\text { Author, } \\
\text { Year }\end{array}$ & Virtual Rehabilitation Intervention & Control Group Intervention \\
\hline Assis, 2014 & $\begin{array}{l}\text { VE: NeuroR augmented reality system, with virtual shoulder } \\
\text { exercises to extend the upper-limb + TAU }\end{array}$ & $\begin{array}{l}\text { TAU: Standard Therapy (NR) } \\
\text { Ancillary: Relaxation session instructed by physiotherapist followed } \\
\text { by shoulder movement using both unaffected and injured arms } \\
\text { assisted by the physiotherapist and also without the physiotherapist } \\
\text { assistance. }\end{array}$ \\
\hline
\end{tabular}

Broeren, VE: Semi-immersive table-top workbench with haptic device 2008

Chen, 2015

Choi, 2014

Crosbie,

2012

da Silva Cameirão, 2011

da Silva

Ribeiro,

2015

Duff, 2010

Gamito,

2015

Givon 2015

Gyuchang

2013

Housman,

2009

$\ln , 2012$

and 3D computer games + TAU

Study 1 - VE: Wii Nintendo games bowling and boxing games + TAUStudy 2 - VE: XaviX games bowling and ladder climbing games + TAU

CG: Wii Nintendo games: swordplay, table tennis, and canoe $+\mathrm{TAU}$

VE: 3D environment system, that included a desktop computer, a head-mounted display unit, a motion tracking system and sensors with tasks focused on reaching and grasping. + TAU

Study 1 - VE: Rehabilitation Gaming System that captures upper limb movements through color detection; two data gloves to capture finger flexure creating a virtual environment where an avatar mimics the movements of the user. Tasks aimed to target speed, range of motion, grasp and release + TAU for 5 weeks. Study 2 - VE: Rehabilitation Gaming System that captures upper limb movements through color detection; two data gloves to capture finger flexure creating a virtual environment where an avatar mimics the movements of the user. Tasks aimed to target speed, range of motion, grasp and release + TAU for 12 weeks.

CG: Wii Nintendo games: tennis, hula-hoop, soccer and boxing. + TAU

VE: Adaptive mixed reality rehabilitation system + TAU

VE: Cognitive stimulation with Serious Games mobile technology, that included several daily live activities e.g. buying items, findings way to the minimarket, finding a virtual character. + TAU

CG: Microsoft Xbox Kinect, Sony PlayStation 2 Eyetoy, Sony PlayStation 3 MOVE, Nintendo Wii Fit and the SeeMe VR systems. + TAU

CG: Xbox Kinect + TAU

Therapy Wilmington Robotic Exoskeleton (T-WREX) a passive (non-robotic) arm that provides support for the arm against gravity and measures arm movement and traces hand grasp as users interact with computer games

VE: Virtual Reality Reflection Therapy program + TAU
TAU: Usual activities at the centre, which included different social activities, creative crafts and physical activities. Ancillary: NA

TAU: At least one hour of physio- and occupational therapy per week.

Ancillary: Two traditional devices, the Curamotion exerciser and the climbing board and bar

\section{TAU: Standard Therapy (NR)}

Ancillary: OT that involved goal-oriented and highly repetitive trainings for that involved composed of stretching and strengthening exercises using full range of motion of the uppe extremity, which was a task-oriented therapy for the ADL, fine motor training, and sensory motor recover

TAU: Standard physiotherapy

Ancillary: Delivered by a physiotherapist, experienced in stroke rehabilitation, and followed a programme of techniques, which included muscle facilitation, stretching exercises, strengthening activities and the inclusion of the more affected upper limb in functional tasks

TAU: Two weekly physiotherapy sessions

Ancillary: involved release or non-specific interactive Wii Nintendo games either intense occupational therapy targeting object displacement, grasp and.

TAU: NR

Ancillary: Conventional physiotherapy that included 10-mintes of upper and lower limb stretching or muscles and trunk, passive, active-assisted, and active-resisted mobilisation of the trunk (10 min), straightening and balance reactions with rapid shifts (10 min), scapular mobilisation (5 min), active or active-assisted upper-limb diagonal movements (15 min) and grasping activities (10 min)

TAU: Standard Therapy (NR) Ancillary: UL therapy: pegboard reaching tasks, bead threading reaching tasks, cone reaching tasks, and $\mathrm{ROM}$ and coordination exercises

TAU: Standard Therapy (NR)

Ancillary: NA

TAU: Standard Therapy (NR)

Ancillary: Participants were then divided into pairs or triads to perform functional activities such as picking up and transferring objects from one side of the room to the other.

TAU: Standard occupational therapy

Ancillary: NA

TAU: Standard Therapy (NR)

Ancillary: Conventional semiautonomous training

TAU: Standard Therapy (NR)Ancillary: The control group received the same treatment as intervention group, but the monitor was off. 
Table 3 Description of the virtual rehabilitation interventions, conventional control group therapies, and additional control treatments, when applicable (Continued)

\begin{tabular}{|c|c|c|}
\hline $\begin{array}{l}\text { First } \\
\text { Author, } \\
\text { Year }\end{array}$ & Virtual Rehabilitation Intervention & Control Group Intervention \\
\hline $\begin{array}{l}\text { Kihoon, } \\
2012\end{array}$ & CG: Interactive Rehabilitation and Exercise System (IREX) + TAU & $\begin{array}{l}\text { TAU: Standard Therapy (NR) } \\
\text { Ancillary: NA }\end{array}$ \\
\hline Kim, 2011 & CG: Interactive Rehabilitation and Exercise System (IREX) + TAU & $\begin{array}{l}\text { TAU: Computer assisted cognitive rehabilitation } \\
\text { Ancillary: NA }\end{array}$ \\
\hline Kim, 2012 & CG: Nintendo Wii Tennis and boxing games & $\begin{array}{l}\text { TAU: Previous therapy (not specified), no therapy at the time of } \\
\text { intervention } \\
\text { Ancillary: NA }\end{array}$ \\
\hline Kiper, 2011 & $\begin{array}{l}\text { VE: Reinforced feedback in virtual environment (RFVE) system } \\
+ \text { TAU }\end{array}$ & $\begin{array}{l}\text { TAU: Standard Therapy (NR) } \\
\text { Ancillary: Additional Standard Therapy (tailored to individual needs) }\end{array}$ \\
\hline Kiper, 2014 & $\begin{array}{l}\text { VE: Reinforced feedback in virtual environment (RFVE) system } \\
+ \text { TAU }\end{array}$ & $\begin{array}{l}\text { TAU: Standard Therapy (NR) } \\
\text { Ancillary: Additional Standard Therapy (tailored to individual needs) }\end{array}$ \\
\hline Kong, 2016 & $\begin{array}{l}\text { CG: Nintendo Wii Tennis, golf, baseball, table tennis, basketball, } \\
\text { cycling, sword play, airplane flight control and boxing games } \\
\text { (Wii Sports and Wii Sports resort packages) + TAU }\end{array}$ & $\begin{array}{l}\text { TAU: Standard Therapy consisted of physical and occupational } \\
\text { therapy one hour a day, Monday to Friday. } \\
\text { Ancillary: NA }\end{array}$ \\
\hline $\begin{array}{l}\text { Kottink, } \\
2014\end{array}$ & VE: The Furball Hunt table-top rehabilitation game + TAU & $\begin{array}{l}\text { Standard therapy tailored to individual needs } \\
\text { Ancillary: Exercises required reaching for targets, such as objects } \\
\text { positioned on a table top, or using specific, non-(electro) } \\
\text { mechanical equipment (bow, pegs in holes, placing disks, etc.) }\end{array}$ \\
\hline Kwon, 2012 & CG: Interactive Rehabilitation and Exercise System (IREX) + TAU & $\begin{array}{l}\text { TAU: Standard physical and occupational therapy } \\
\text { Ancillary: NA }\end{array}$ \\
\hline Lee, 2013 & CG: Xbox Kinect + TAU & $\begin{array}{l}\text { TAU: Standard occupational therapy } \\
\text { Ancillary: NA }\end{array}$ \\
\hline Levin, 2012 & $\begin{array}{l}\text { VE: Virtual games and a virtual supermarket (e.g., Birds \& Balls, } \\
\text { Soccer, Volleyball, VMall) + TAU }\end{array}$ & $\begin{array}{l}\text { TAU: NR } \\
\text { Ancillary: Occupational therapy, including exercises involving } \\
\text { reaching for and holding cones, cups, and other objects in all } \\
\text { motion planes with and without external loading }\end{array}$ \\
\hline Piron, 2009 & $\begin{array}{l}\text { VE: Therapist telerehabilitation equipment }\left(V R R S \text { net }{ }^{\oplus}\right) \text {, } \\
\text { participant can observe his/her movement on the screen } \\
\text { (augmented feedback), and observe the correct trajectory } \\
\text { pre-recorded in the virtual scene (virtual teacher) + TAU }\end{array}$ & $\begin{array}{l}\text { TAU: NR } \\
\text { Ancillary: Conventional physical therapy, with patients performing } \\
\text { specific exercises (e.g. touching different targets arranged in a } \\
\text { horizontal plane in front of them) with a strategy of progressive } \\
\text { complexity. }\end{array}$ \\
\hline Piron, 2010 & $\begin{array}{l}\text { VE: Reinforced feedback in a virtual environment (RFVE) } \\
\text { system + TAU }\end{array}$ & $\begin{array}{l}\text { TAU: Conventional physical therapy } \\
\text { Ancillary: The CT program was based on Bobath principles. The } \\
\text { patients performed specific exercises with the upper limb with } \\
\text { progressive complexity. First, the patients were asked to control } \\
\text { isolated motions without postural control; subsequently, postural } \\
\text { control was included; and finally, complex motion with postural } \\
\text { control was practiced. }\end{array}$ \\
\hline $\begin{array}{l}\text { Saposnik, } \\
2016\end{array}$ & $\begin{array}{l}\text { CG: Nintendo Wii, Wii sports and Game Party } 3 \text { packages } \\
+ \text { TAU }\end{array}$ & $\begin{array}{l}\text { TAU: Standard Therapy (NR) } \\
\text { Ancillary: Recreational computer-generated activities } \\
\text { (passive control) }\end{array}$ \\
\hline Shin, 2014 & VE: The RehabMaster game-based system + TAU & $\begin{array}{l}\text { TAU: Standard Therapy (NR) } \\
\text { Ancillary: Occupational therapy }\end{array}$ \\
\hline Shin, 2015 & VE: The RehabMaster game-based system + TAU & $\begin{array}{l}\text { TAU: Standard Therapy (NR) } \\
\text { Ancillary: Occupational therapy }\end{array}$ \\
\hline Sin, 2013 & CG: Xbox Kinect Microsoft + TAU & $\begin{array}{l}\text { TAU: Standard Therapy (NR) } \\
\text { Ancillary: NA }\end{array}$ \\
\hline $\begin{array}{l}\text { Standen, } \\
2017\end{array}$ & $\begin{array}{l}\text { VE: Home-based VR system with three games (Spacerace, } \\
\text { Spongeball, Balloonpop) }\end{array}$ & $\begin{array}{l}\text { TAU: Previous therapy, no therapy at the time of intervention } \\
\text { Ancillary: NA }\end{array}$ \\
\hline $\begin{array}{l}\text { Yavuzer, } \\
2008\end{array}$ & $\begin{array}{l}\text { CG: Playstation EyeToy games (Kung-foo, goal attack, } \\
\text { MrChef, Dig and HomeRun) + TAU }\end{array}$ & $\begin{array}{l}\text { TAU: Physiotherapy, occupational therapy, speech therapy } \\
\text { (if needed) } \\
\text { Ancillary: "Sham therapy" (not specified, passive control) }\end{array}$ \\
\hline Yin, 2014 & VE: VE of a local supermarket setting + TAU & $\begin{array}{l}\text { TAU: Standard Therapy (NR) } \\
\text { Ancillary: NA }\end{array}$ \\
\hline
\end{tabular}

Note: CG Commercial Gaming, NA Not Applicable, NR Not Reported, TAU Treatment as Usual, VE Virtual Environment 
to individual needs $[77,79,93]$ (see Table 3 ). In contrast, 12 studies utilized "passive" control groups that received no additional intervention beyond treatment as usual.

\section{Dose and session scheduling}

For all VR approaches combined, the mean overall Dose was $685 \mathrm{~min}(S D=355$, range $200-1440 \mathrm{~min})$, with a mean Daily Intensity of $42 \mathrm{~min}(S D=15$, median 30, range 20-60 $\mathrm{min}$ ) and Weekly Intensity of $153.9 \mathrm{~min}$ $(S D=80.38$, median $=135$, range 60-800). The mean Frequency was three sessions a week (range one-five sessions), and the median Duration was 18 sessions (range, 4-36 sessions).

\section{ICF-WHO outcomes}

Twenty-seven studies reported Body Structure/Function level outcomes, with the Fugl-Meyer Assessment-Upper Extremity (FMA-UE) as the most common instrument (21 studies). An additional study [95] utilized the FMAUE to classify baseline participant characteristics but did not include it as an outcome measure. Twenty-nine studies reported Activity level outcomes, most commonly using the Box and Blocks Test (seven studies), Functional Independent Measure (eight studies), and Barthel Index (six studies). Participation level outcomes were reported by five studies, most often utilizing the Motor Activity Log instrument (four studies). Only four studies [53, 54, 91, 97] reported data on cognitive outcomes (see Table 4), each of these studies reported data on multiple cognitive outcomes, and all of these were included in the analyses (Table 5).

\section{Risk of bias}

The methodological quality of included studies was generally high (see Table 4), with an average PEDro total score of $7.06(S D=1.26$, range 5-9). Eligibility criteria were specified in all studies, and all but one study [83] specified random allocation of participants. However, despite more rigorously focusing only on RCT designs, However, despite more rigorously focusing only on RCT designs, four [21, 80, 85, 92] of the included studies were rated only fair quality, due to the omission of concealed allocation, blinding, and intention to treat analyses. In addition, the Egger's intercept value for all outcomes combined was 1.23, $p=0.02$ (two-tailed), suggesting pronounced asymmetry and an increased likelihood that smaller studied tended to report larger than average effects [98]. To minimize the risk of publication bias all reported effect size outcomes were based on a randomeffects model to give more weight to larger trials [99].

\section{Main effects of VR after stroke}

For all outcomes combined (see Fig. 3 and Additional file 1: Figure S1), the average effect size for VR interventions was small to medium ( $g=0.46$; 95\% CI: 0.33-0.59, $p<0.01)$, with significant benefit of VR compared to CT. The overall fail-safe $N$ was high at 439 , and heterogeneity minimal $\left(I^{2}=0 \%\right)$, suggesting a robust finding. Both VE and CG approaches were significantly more effective than $\mathrm{CT}$, with an average small effect size for CG $(g=0.33 ; 95 \% \mathrm{CI}$ : $0.14-0.51, p<0.01)$, and an average medium effect size for VE interventions $(g=0.58$; 95\% CI: $0.41-0.76$, $p<0.01$ ). Moderator analysis confirmed the difference between VE and CG-based approaches was statistically significant $[Q(1)=3.96, p=0.047]$.

The average effect size for cognitive outcomes was small but significant $(g=0.45,95 \%$ CI: $0.02-0.88, p$ $=0.04)$. Heterogeneity between studies was minimal $\left(I^{2}=14.69 \%\right)$, but the fail-safe $N$ was only 2 , suggesting a tenuous finding. For upper-limb motor and functional outcomes, data was examined at each of the three ICF-WHO levels (see Fig. 3 and Additional file 2: Figure S2). Small overall to medium effects were observed on Body Structures/ Function ( $g=0.41 ; 95 \%$ CI: $0.28-0.55 ; p<0.01)$ and Activity outcomes ( $g=0.47$; $95 \%$ CI: $0.34-0.60, p<$ 0.01 ), while Participation outcomes were non significant $(g=0.38$; 95\% CI: $-0.29-1.04, p=0.27)$.

\section{Moderator analysis}

Moderator analysis (see Fig. 4) found no significant difference in the overall outcomes of interventions that utilized an active or passive control group $[Q(1)=0.05$, $p=0.83$, and between moderate and high quality studies $[Q(1)=0.001, p=0.98]$, and between studies with low and high sample size $[Q(1)=0.67, p=0.41]$. Moreover, there was no significant difference in overall outcomes for patients receiving VR during either the sub-acute or chronic stage $[Q(1)=2.39, p=0.12]$, and between interventions that focused specifically on hand function or overall upper-limb function $[Q(1)=2.82, p=0.09]$.

Different levels of dose (high, medium, low) had no significant effect on the overall effect $[Q(2)=2.22, p=$ $0.33]$. Variations in daily intensity $[Q(1)=0.16, p=0.70]$, frequency $[Q(1)=0.67, p=0.71]$, weekly intensity $[Q(1)$ $=0.03, p=0.85]$ and duration $[Q(1)=2.77, p=0.10]$ also had no significant impact.

Meaningful comparisons could not be performed between different levels of severity (determined using gold standard FMA-UE outcome measure); there was only a single study that used a group of mild severity [82]. The larger (moderate-severe) group clustered tightly around a mean severity of 34.9 (SD: 8.9). When the mild severity study [82] was pulled-out from the overall analysis, the 
Table 4 Outcome measures included in the data analysis

\begin{tabular}{|c|c|c|c|c|c|c|c|c|c|c|}
\hline \multirow[b]{2}{*}{$\begin{array}{l}\text { First } \\
\text { Author, } \\
\text { Year }\end{array}$} & \multicolumn{3}{|c|}{ Body Structure and Function } & \multicolumn{5}{|c|}{ Activity } & \multirow[t]{2}{*}{ Participation } & \multirow[t]{2}{*}{ Cognitive } \\
\hline & $\begin{array}{l}\text { FMA- } \\
\text { UE }\end{array}$ & MAS & Other outcomes & $\overline{\mathrm{BBT}}$ & ARAT & FIM & $\mathrm{Bl}$ & Other outcomes & & \\
\hline Assis, 2014 & $\checkmark$ & & & & & & & & & \\
\hline $\begin{array}{l}\text { Broeren, } \\
2008\end{array}$ & & & & $\checkmark$ & & & & & & \\
\hline $\begin{array}{l}\text { Chen, } \\
2015\end{array}$ & $\checkmark$ & & & $\checkmark$ & & $\checkmark$ & & & & \\
\hline Choi, 2014 & $\checkmark$ & & MFT; Grip Strength & $\checkmark$ & & & $\checkmark$ & & & $\begin{array}{l}\text { A-CPT Correct Detection, Reaction } \\
\text { Time, Commission Error; V-CPT } \\
\text { Correct Detection, Reaction Time }\end{array}$ \\
\hline $\begin{array}{l}\text { Crosbie, } \\
2012\end{array}$ & & & & & $\checkmark$ & & & $\mathrm{Ml}$ & & \\
\hline $\begin{array}{l}\text { da Silva } \\
\text { Cameirão, } \\
2011\end{array}$ & $\checkmark$ & & $\begin{array}{l}\text { FMA arm; FMA hand/ } \\
\text { wrist }\end{array}$ & & & & $\checkmark$ & CAHAl; MI & & \\
\hline $\begin{array}{l}\text { da Silva } \\
\text { Ribeiro, } \\
2015\end{array}$ & $\checkmark$ & & $\begin{array}{l}\text { FMA Overall, Physical } \\
\text { Functioning (overall } \\
\text { motor functioning }\end{array}$ & & & & & $\begin{array}{l}\text { SF-36 Social Aspects, } \\
\text { Vitality }\end{array}$ & & \\
\hline Duff, 2010 & $\checkmark$ & & $\begin{array}{l}\text { FMA range of } \\
\text { motion, pain, } \\
\text { sensation }\end{array}$ & & & & & $\begin{array}{l}\text { SIS; WMFT total; } \\
\text { WMFT time. }\end{array}$ & $\begin{array}{l}\text { MAL Amount of } \\
\text { Use, Quality of } \\
\text { Movement }\end{array}$ & \\
\hline $\begin{array}{l}\text { Gamito, } \\
2015\end{array}$ & & & & & & & & & & TP; WMS III \\
\hline $\begin{array}{l}\text { Givon } \\
2015\end{array}$ & & & $\begin{array}{l}\text { Grip Strength } \\
\text { Weaker Hand; Gait } \\
\text { Speed (Motor Overall) }\end{array}$ & & $\checkmark$ & & & & & \\
\hline $\begin{array}{l}\text { Housman, } \\
2009\end{array}$ & $\checkmark$ & & Grip strength & & & & & $\begin{array}{l}\text { Racho level (UL); } \\
\text { Racho Speed (UL) }\end{array}$ & $\begin{array}{l}\text { MAL Amount of } \\
\text { Use, Quality of } \\
\text { Movement }\end{array}$ & \\
\hline In, 2012 & $\checkmark$ & $\checkmark$ & MFT & $\checkmark$ & & & & JHFT & & \\
\hline $\begin{array}{l}\text { Kihoon, } \\
2012\end{array}$ & & & & & & & & $\begin{array}{l}\text { WMFT total, hand, } \\
\text { arm }\end{array}$ & & $\begin{array}{l}\text { MVPT Reaction time in seconds; } \\
\text { MVPT score; Visual discrimination; } \\
\text { Visual memory; visual closure }\end{array}$ \\
\hline Kim, 2011 & & & & & & & $\checkmark$ & $\mathrm{Ml}$ & & $\begin{array}{l}\text { VCPT; ACPT; } \\
\text { WCW; CCW; } \\
\text { FDST; BDST; FVST; BVST; } \\
\text { ViLT; VeLT; TMT-A; TOL }\end{array}$ \\
\hline Kim, 2012 & & $\checkmark$ & PASS Motor overall & & & $\checkmark$ & & & & \\
\hline Kiper, 2011 & $\checkmark$ & $\checkmark$ & & & & $\checkmark$ & & & & \\
\hline Kiper, 2014 & $\checkmark$ & & $\begin{array}{l}\text { Mean Duration of } \\
\text { Movement - time; } \\
\text { peak; speed }\end{array}$ & & & $\checkmark$ & & & & \\
\hline $\begin{array}{l}\text { Kong, } \\
2016\end{array}$ & $\checkmark$ & & & & $\checkmark$ & $\checkmark$ & & & & \\
\hline $\begin{array}{l}\text { Kottink, } \\
2014\end{array}$ & $\checkmark$ & & & & $\checkmark$ & & & & & \\
\hline $\begin{array}{l}\text { Kwon, } \\
2012\end{array}$ & $\checkmark$ & & $\begin{array}{l}\text { MFT; FMA arm, } \\
\text { hand, wrist }\end{array}$ & & & & $\checkmark$ & K-MBI Self Care & & \\
\hline Lee, 2013 & $\checkmark$ & & $\begin{array}{l}\text { MMT Muscle } \\
\text { Strength; }\end{array}$ & & & $\checkmark$ & & & & \\
\hline $\begin{array}{l}\text { Levin, } \\
2012\end{array}$ & $\checkmark$ & & RPSS & $\checkmark$ & & & & WMFT & $\begin{array}{l}\text { MAL Amount of } \\
\text { Use, Quality of } \\
\text { Movement }\end{array}$ & \\
\hline
\end{tabular}


Table 4 Outcome measures included in the data analysis (Continued)

\begin{tabular}{|c|c|c|c|c|c|c|c|c|c|c|}
\hline \multirow[b]{2}{*}{$\begin{array}{l}\text { First } \\
\text { Author, } \\
\text { Year }\end{array}$} & \multicolumn{3}{|c|}{ Body Structure and Function } & \multicolumn{5}{|c|}{ Activity } & \multirow[t]{2}{*}{ Participation } & \multirow[t]{2}{*}{ Cognitive } \\
\hline & $\begin{array}{l}\text { FMA- } \\
\text { UE }\end{array}$ & MAS & Other outcomes & $\overline{\mathrm{BBT}}$ & ARAT & $\mathrm{FIM}$ & $\mathrm{Bl}$ & Other outcomes & & \\
\hline $\begin{array}{l}\text { Piron, } \\
2009\end{array}$ & $\checkmark$ & $\checkmark$ & & & & & & & & \\
\hline $\begin{array}{l}\text { Piron, } \\
2010\end{array}$ & $\checkmark$ & & & & & $\checkmark$ & & & & \\
\hline $\begin{array}{l}\text { Saposnik, } \\
2016\end{array}$ & & & & $\checkmark$ & & $\checkmark$ & $\checkmark$ & WMFT & & \\
\hline Shin, 2014 & $\checkmark$ & & & & & & $\checkmark$ & & & \\
\hline Shin, 2015 & $\checkmark$ & & & & & & & $\begin{array}{l}\text { SF-36 Role limitation } \\
\text { due to physical } \\
\text { problems; SF-36 } \\
\text { Vitality }\end{array}$ & & \\
\hline Sin, 2013 & $\checkmark$ & & & $\checkmark$ & & & & & & \\
\hline $\begin{array}{l}\text { Standen, } \\
2017\end{array}$ & & & NHPT & & & & & NEADL; WMFT & $\begin{array}{l}\text { MAL Amount of } \\
\text { Use, Quality of } \\
\text { Movement; } \\
\text { MAL-activities }\end{array}$ & \\
\hline $\begin{array}{l}\text { Yavuzer, } \\
2008\end{array}$ & & & & & & & & $\begin{array}{l}\text { Brunnstorm stages } \\
\text { hand, Upper } \\
\text { Extremity; FIM Self } \\
\text { Care }\end{array}$ & & \\
\hline Yin, 2014 & $\checkmark$ & $\checkmark$ & & & $\checkmark$ & & & & $\begin{array}{l}\text { MAL Amount of } \\
\text { Use, Quality of } \\
\text { Movement }\end{array}$ & \\
\hline
\end{tabular}

Note: ACPT Auditory continuous performance test, AF Augmented Feedback, ARAT Action Research Arm Test, AS Ashworth scale, BDST Backward digit span test, BVST Backward visual span test, BI Barthel Index, BBT Box and Blocks test, CCW Color of word in word-color test, CAHAI Chedoke Arm and Hand Activity Inventory, CSI Composite Spasticity Index, FMA Fugl-Meyer Assessment, FMA-UE Fugl-Meyer Assessment-Upper Extremity FDST: Forward digit span test, FVST Forward visual span test, JHFT Jebsen Hand Function Test, K-MBI Korean version of the Modified Barthel Index, MAS: Modified Ashworth Scale, MAL Motor Activity Log, MFT Manual Function Test, MI Motricity Index, TPT Toulouse-Pieron Test, VCPT Visual continuous performance test, ViLT Visual learning test, VeLT Verbal learning test, TMT-A Trail Making Test-A, TOL Tower of London test, QOM Quality of Movement, RPSS Reaching Performance Scale for Stroke, WMFT Wolf Motor Function Test, WMS-III Wechsler Memory Scale Third Edition, WCW Word in word-color test

overall effect for all outcomes combined remained small $(g=0.47 ; 95 \%$ CI: $0.34-0.60, p<0.01)$, with significant benefit of VR compared with CT.

On the basis of the statistically significant advantage for $\mathrm{VE}$ approaches relative to CG designs, treatment effects for VE-based rehabilitation alone were also analyzed at each ICF-WHO level (see Fig. 5). There was a medium effect overall on Body Structure/Function $(g=0.54 ; 95 \%$ CI: $0.35-0.73 ; p<0.01)$, and a medium to large effect on Activity ( $g=0.62 ; 95 \% \mathrm{CI}$ : $0.43-0.81, p<0.01)$. The overall effect on Participation was unchanged as no $\mathrm{CG}$ approaches examined outcomes in this ICF-WHO domain. Within-group heterogeneity was minimal for Activity $\left(I^{2}=0 \%\right)$ and Body Function $\left(I^{2}=0 \%\right)$ outcomes, and large for Participation outcomes $\left(I^{2}=65 \%\right)$.

\section{Follow-up data}

Twelve studies also included follow-up data: six studies reassessed outcomes four to six weeks after intervention [44, $76,79,86,94,96]$ and six studies re-assessed outcomes eight to 26 weeks later $[74,87,88,90,93,95]$. Both CG [74,
86, 88, 95] and VE [44, 76, 79, 87, 90, 93, 94, 96] approaches, and sub-acute $[44,74,76,86]$ and chronic $[79$, 87, 88, 90, 93-96] populations were represented (see Fig. 6). There was no significant difference in treatment effect $[Q(2)=0.35, p=0.72]$ between the four to six week follow-up $(g=0.36, p=0.02)$, the eight to 26 week follow-up $(g=0.58, p<0.01)$, and the overall effect of VR observed immediately following intervention $(g=0.46, p<$ 0.01). Differences between CG and VE approaches were not statistically significant at either follow-up [4-6 weeks: $Q(1)$ $=2.03, p=0.15$; 8-26 weeks: $Q(1)=0.10, p=0.76]$. Overall, small to medium effects for both Body Structure/Function and Activity level outcomes were observed at both the four to six week and the eight to 26 week follow-ups. Only three studies examined Participation outcomes at a follow-up $[44,87,90]$, which were small, and non-significant $(p=$ 0.48 ), in keeping with the pre-post findings. No studies examined cognitive outcomes at follow-up. Consistent with the pre-post data analysis, treatment effects did not vary as a function of the implementation parameters (i.e. dose, daily intensity, weekly intensity, frequency, duration), or recovery stage (i.e. sub-acute vs. chronic). 
Table 5 PEDro Scale risk of bias ratings for the included studies

\begin{tabular}{|c|c|c|c|c|c|c|c|c|c|c|c|c|}
\hline First Author, Year & $\mathrm{C} 1$ & $\mathrm{C} 2$ & $\mathrm{C} 3$ & C4 & C5 & C6 & $\mathrm{C} 7$ & $\mathrm{C} 8$ & C9 & $\mathrm{C} 10$ & $\mathrm{C} 11$ & TOTAL \\
\hline Assis, 2014 & 1 & 1 & 0 & 0 & 0 & 0 & 0 & 1 & 1 & 1 & 1 & 6 \\
\hline Broeren, 2008 & 1 & 1 & 0 & 1 & 0 & 0 & 0 & 1 & 0 & 0 & 1 & 5 \\
\hline Chen, 2015 & 1 & 0 & 0 & 1 & 0 & 0 & 1 & 1 & 1 & 1 & 1 & 7 \\
\hline Choi, 2014 & 1 & 1 & 1 & 1 & 0 & 0 & 1 & 1 & 1 & 1 & 1 & 9 \\
\hline Crosbie, 2012 & 1 & 1 & 1 & 1 & 0 & 0 & 1 & 1 & 1 & 1 & 1 & 9 \\
\hline da Silva Cameirão, 2011 & 1 & 1 & 0 & 0 & 0 & 0 & 1 & 0 & 0 & 1 & 1 & 5 \\
\hline da Silva Ribeiro, 2015 & 1 & 1 & 1 & 1 & 0 & 0 & 1 & 1 & 0 & 1 & 1 & 8 \\
\hline Duff, 2010 & 1 & 1 & 0 & 1 & 0 & 0 & 1 & 0 & 0 & 1 & 1 & 6 \\
\hline Gamito, 2015 & 1 & 1 & 0 & 0 & 0 & 0 & 0 & 1 & 1 & 1 & 1 & 6 \\
\hline Givon, 2015 & 1 & 1 & 0 & 1 & 0 & 0 & 1 & 1 & 1 & 1 & 1 & 8 \\
\hline Housman, 2009 & 1 & 1 & 1 & 1 & 0 & 0 & 1 & 1 & 0 & 1 & 1 & 8 \\
\hline In, 2012 & 1 & 1 & 0 & 1 & 0 & 0 & 0 & 0 & 0 & 1 & 1 & 5 \\
\hline Kihoon, 2012 & 1 & 1 & 0 & 1 & 0 & 0 & 0 & 1 & 0 & 1 & 1 & 6 \\
\hline Kim, 2011 & 1 & 1 & 0 & 1 & 0 & 0 & 0 & 1 & 1 & 1 & 1 & 7 \\
\hline Kim, 2012 & 1 & 1 & 0 & 1 & 0 & 0 & 0 & 0 & 0 & 1 & 1 & 5 \\
\hline Kiper, 2011 & 1 & 1 & 0 & 1 & 0 & 0 & 0 & 1 & 1 & 1 & 1 & 7 \\
\hline Kiper, 2014 & 1 & 1 & 0 & 1 & 0 & 0 & 0 & 1 & 1 & 1 & 1 & 7 \\
\hline Kong, 2016 & 1 & 1 & 1 & 1 & 0 & 0 & 1 & 1 & 1 & 1 & 1 & 9 \\
\hline Kottink, 2014 & 1 & 1 & 0 & 1 & 0 & 0 & 1 & 1 & 0 & 1 & 1 & 7 \\
\hline Kwon, 2012 & 1 & 1 & 0 & 1 & 1 & 0 & 1 & 1 & 1 & 1 & 1 & 9 \\
\hline Lee, 2013 & 1 & 1 & 0 & 0 & 0 & 0 & 0 & 1 & 1 & 1 & 1 & 6 \\
\hline Levin, 2012 & 1 & 1 & 0 & 1 & 0 & 0 & 1 & 1 & 0 & 1 & 1 & 7 \\
\hline Piron, 2009 & 1 & 1 & 1 & 0 & 0 & 0 & 1 & 1 & 1 & 1 & 1 & 8 \\
\hline Piron, 2010 & 1 & 1 & 1 & 0 & 0 & 0 & 1 & 1 & 1 & 1 & 1 & 8 \\
\hline Saposnik, 2016 & 1 & 1 & 0 & 1 & 0 & 0 & 1 & 1 & 1 & 1 & 1 & 8 \\
\hline Shin, 2014 & 1 & 1 & 0 & 1 & 0 & 0 & 1 & 1 & 0 & 1 & 1 & 7 \\
\hline Shin, 2015 & 1 & 1 & 0 & 1 & 0 & 0 & 1 & 1 & 0 & 1 & 1 & 7 \\
\hline Sin, 2013 & 1 & 1 & 0 & 1 & 0 & 0 & 1 & 1 & 0 & 1 & 1 & 7 \\
\hline Standen, 2017 & 1 & 1 & 1 & 1 & 0 & 0 & 0 & 1 & 0 & 1 & 1 & 7 \\
\hline Yavuzer, 2008 & 1 & 1 & 1 & 1 & 0 & 0 & 1 & 1 & 1 & 1 & 1 & 9 \\
\hline Yin 2014 & 1 & 1 & 1 & 1 & 0 & 0 & 0 & 0 & 0 & 1 & 1 & 6 \\
\hline
\end{tabular}

Note: " 1 " indicates a study met the criteria, " 0 " indicates there was not enough information to make an assessment or the criterion was not met. $\mathrm{C} 1$ = Eligibility criteria were specified. C2 = Subjects were randomly allocated to groups (in a crossover study, subjects were randomly allocated an order in which treatments were received). C $3=$ Allocation was concealed. $\mathrm{C} 4=$ The groups were similar at baseline regarding the most important prognostic indicators. $\mathrm{C} 5=$ There was blinding of all subjects. $\mathrm{C} 6=$ There was blinding of all therapists who administered the study. $\mathrm{C} 7=$ There was blinding of all assessors who measured at least one key outcome. C8 = Measures of at least one key outcome were obtained from more than $85 \%$ of the subjects initially allocated to groups. $C 9=$ All subjects for whom outcome measures were available received the treatment or control condition as allocated or, where this was not the case, data for at least one key outcome was analyzed by "intention to treat." C10 = The results of between-group statistical comparisons are reported for at least one key outcome. $\mathrm{C} 11=$ The study provides both point measures and measures of variability for at least one key outcome

\section{Discussion}

VR is an engaging form of therapy for stroke [19] and suggested to enhance motor, functional, and cognitive performance $[11,19,46,54]$, whether delivered via VE $[11,46]$ or CG [11]. While recent reviews of VR therapy have shown improvement in upper limb function, superior to conventional physical therapy $[11,19,46,58,62]$, we know little of treatment effects across all ICF-WHO levels and how outcomes vary along different implementation parameters and design factors $[19,46]$, resulting in uncertainties about the optimal training protocol that affords the greatest efficacy. The aim of this study was to address these gaps in understanding by 


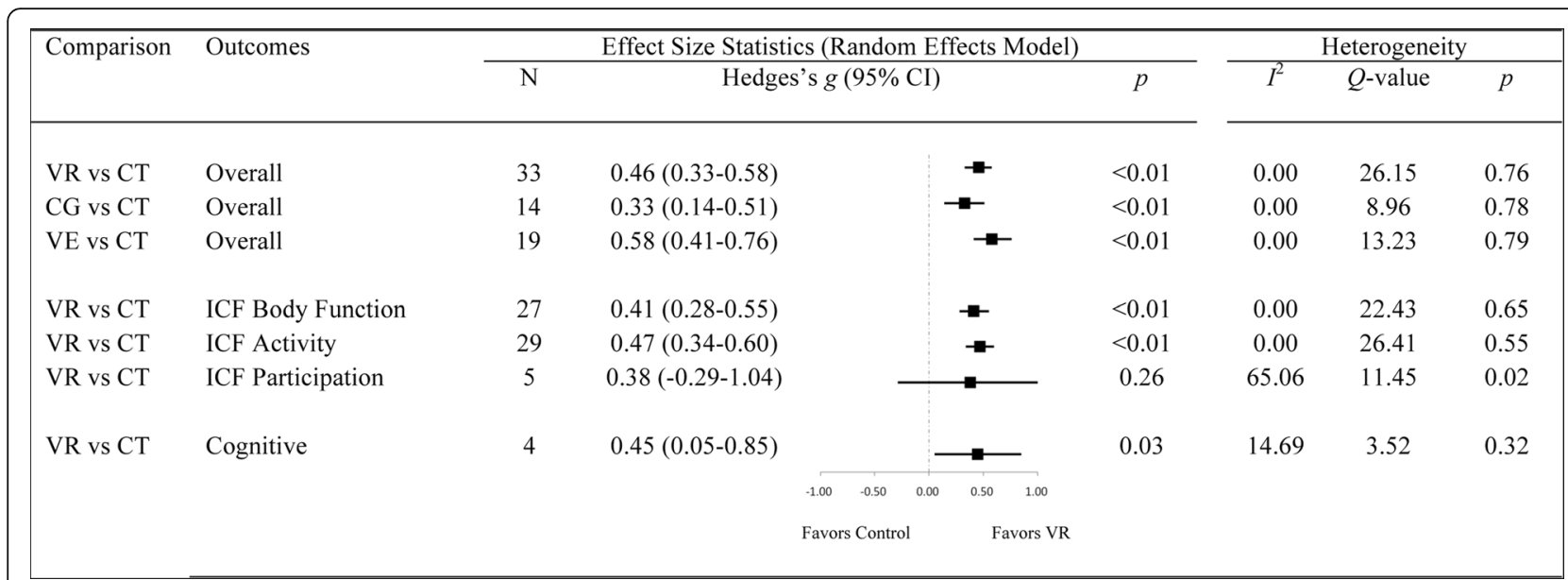

Fig. 3 Forest plot showing the main effect-sizes of Virtual Rehabilitation after stroke on the motor, functional, and cognitive outcomes combined; the three levels of the International Classification of Functioning (Body Function outcomes included Fugl-Meyer Assessment-Upper Extremity and Modified Ashworth Scale; Activity outcomes included Box and Blocks Test; Participation outcomes included Motor Activity Log and Quality of Movement); and cognitive outcomes using the random-effects model. Notes: CG: Computerized Gaming; Cl: Confidence Intervals; CT: Conventional Treatment; ICF: International Classifacation of Functioning; VE: Virtual Environment; VR: Virtual Rehabilitation

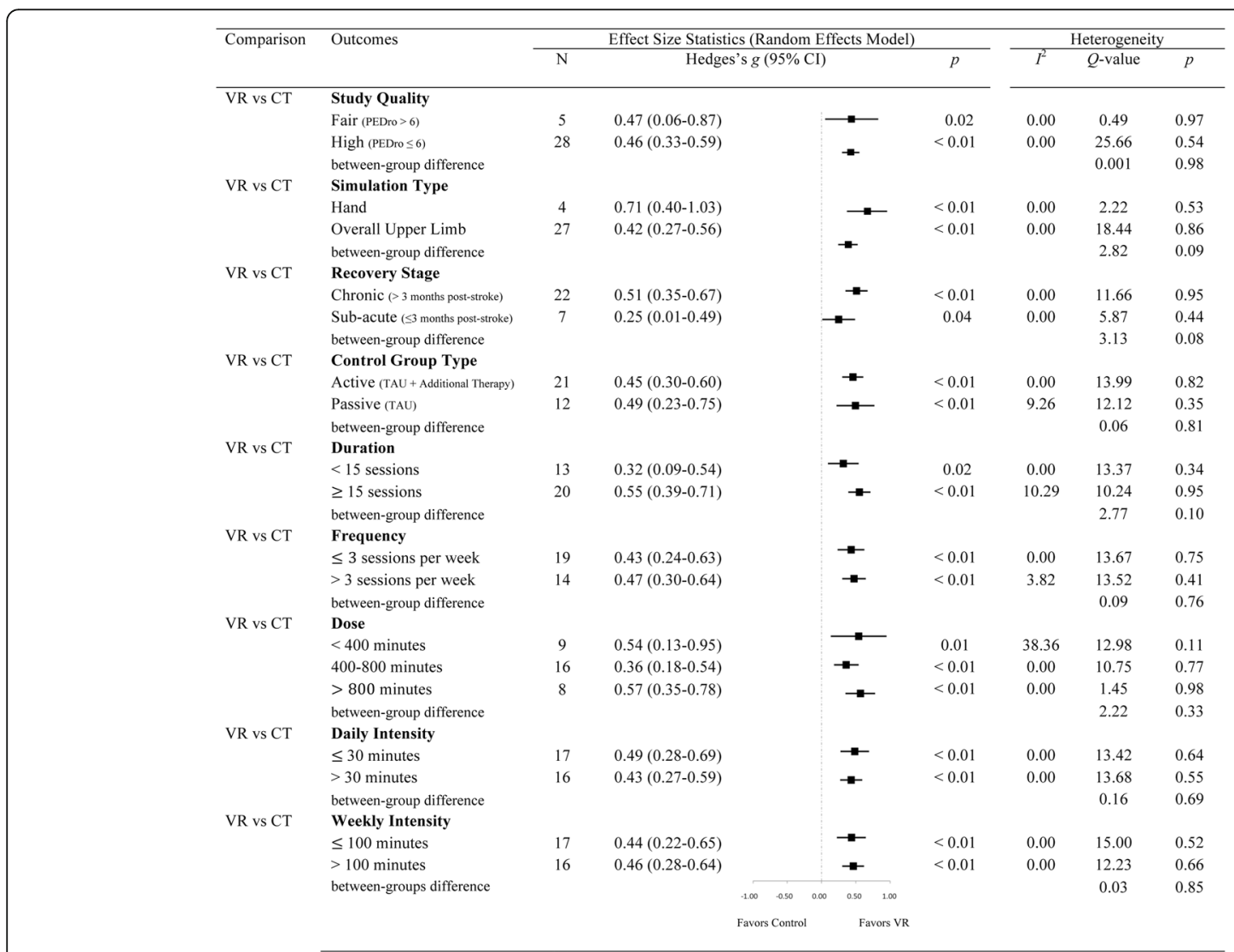

Fig. 4 Forest plot showing the main moderator analyses of Virtual Rehabilitation outcomes after stroke using the random-effects model. Note: AR: Additional Rehabilitation; Cl: Confidence Intervals; CT: Conventional Treatment; TAU; Treatment As Usual; VR: Virtual Rehabilitation 


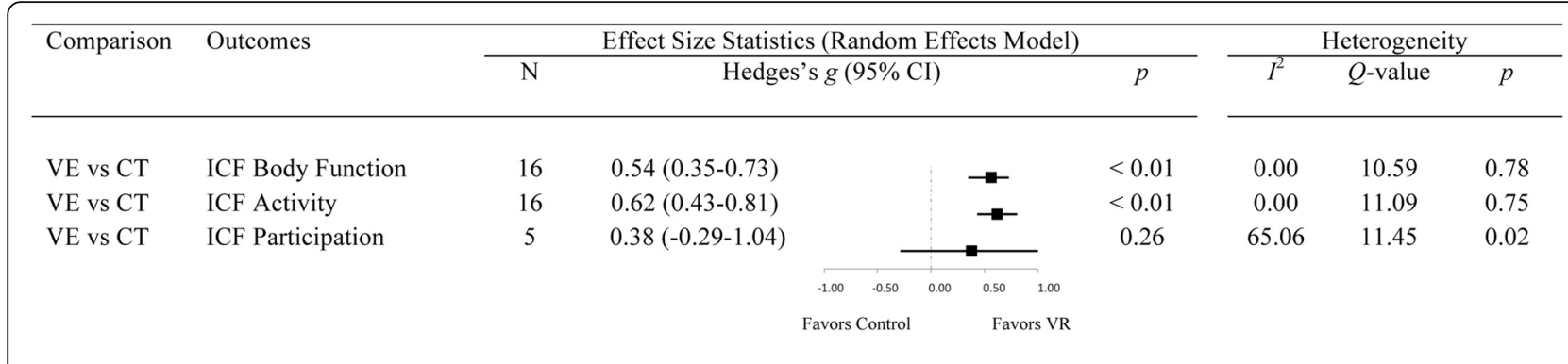

Fig. 5 Forest plot showing the main effect-sizes of Virtual Environment therapy after stroke on the three levels of the International Classification of Functioning using the random-effects model. Body Function outcomes included Fugl-Meyer Assessment-Upper Extremity and Modified Ashworth Scale; Activity outcomes included Box and Blocks Test; Participation outcomes included Motor Activity Log and Quality of Movement. Note: Cl; Confidence Intervals; CT: Conventional Treatment; ICF: International Classifacation of Functioning; VE: Virtual Environment

analyzing the current evidence base on VR of upperlimb and cognitive function in stroke, in a combined systematic review and meta-analysis.

Overall, the current review of 33 RCTs found that when compared with conventional therapies, VR interventions produced a small to medium overall effect $(g=0.46)$, above and beyond conventional physical rehabilitation. Specifically, small to medium effects were observed on Body Structure/Function ( $g$ $=0.41)$ and Activity outcomes $(g=0.47)$, while Participation outcomes $(g=0.38)$ were highly variable $\left(I^{2}=65 \%\right)$ but overall non-significant $(p=0.26)$. A small to medium effect on cognitive outcomes was shown $(g=0.41)$, albeit based on only four studies. Intriguingly, the effect of VR was not moderated by dose-related parameters, and no moderator effects for chronicity were evident. These results are discussed in detail below.

\section{Overall effectiveness of virtual rehabilitation}

The extent of motor recovery after conventional stroke rehabilitation is often "modest" $[100,101]$ with no significant advantage between different approaches $[100,102]$. When compared with these conventional interventions (including occupational therapy and physiotherapy), the current meta-analysis showed an additional small to medium treatment effect in favor of VR, above and beyond the gains of treatment as usual. The magnitude of this benefit was comparable to that shown in earlier quantitative reviews $[11,19$, $46,65]$ and reflects an important advance in rehabilitation outcomes. Other attempts to identify novel

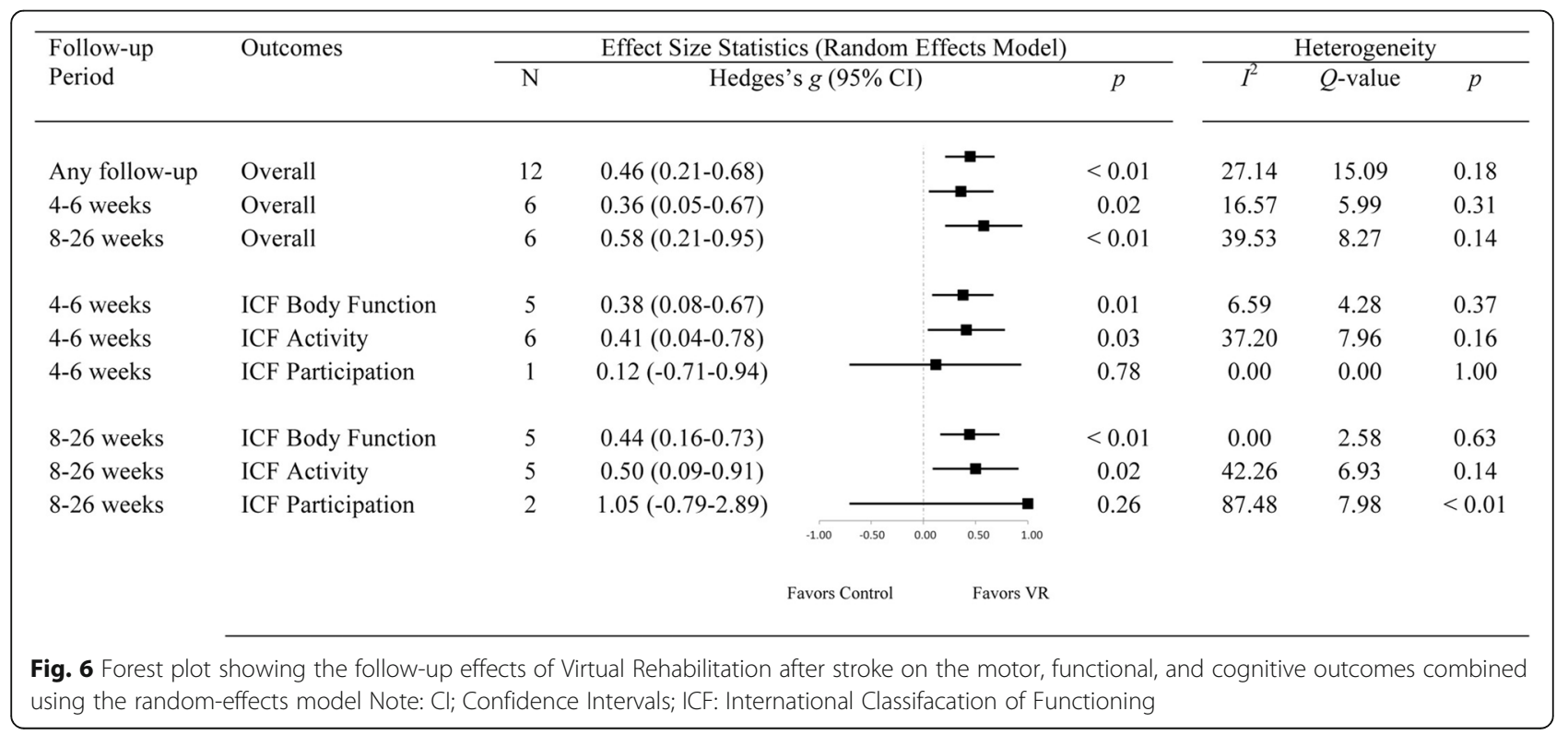


adjunctive therapies to boost the effects of conventional rehabilitation have been less successful. For example, a review of robotic-assisted therapy for stroke patients with upper-limb impairment [103] showed no significant difference between intensive conventional therapy and robotic-assisted therapy groups in terms of motor recovery, activities of daily living, strength, and motor control.

\section{Virtual environment versus commercial gaming systems}

The current review evaluated two main types of VR interventions: purpose-designed $\mathrm{VE}$ platforms were examined in 19 studies and commercially available CG systems in 14. Previous reviews have also examined the separate impact of these two types of intervention, but with too few CG studies to make any firm conclusions about relative efficacy [11, 46]. In contrast to the previous two major reviews, which included only $17 \%$ [11] and 22\% [46] CG-based studies, almost half $(42 \%)$ of the studies included in the current review were CG-based interventions, suggesting a growing interest in off-the-shelf solutions.

In the current review, both VE and CG intervention types were significantly superior to conventional therapies, with medium effect sizes observed for VE platforms $(g=0.59)$ and small effects for CG systems $(g=0.33)$. This difference between VR approaches was statistically significant, and suggests that while both VE and CG systems afford good training effects overall, VE-based systems are somewhat superior [46]. This finding supports the value of customizing rehabilitation tasks according to the clinical needs and capacities of patients. Consistent with previous reviews $[11,46]$ the positive effect of $\mathrm{VE}$ approaches was observed mainly on outcomes at the Body Structure/Function and Activity levels of the ICF, which is discussed in the next section.

\section{Virtual rehabilitation outcomes by domains of function}

Over 50 different outcome measures were used by studies included in the current review, underlining the importance of standardized classification using the ICF-WHO [58, 104]. For outcomes at the Body Structure/Function and Activity levels, effects sizes for VR (VE and CG combined) were significant (0.41 and 0.47 , respectively). Effects at these levels of the ICFWHO were more pronounced, however, when VE systems were considered separately: 0.54 and 0.62 for Body Structure/Function and Activity, respectively, compared with 0.27 and 0.32 for CG systems. The results for VE approaches were comparable to previously reported effect sizes at the Body Function ( $g=0.48)$, and Activity $(g=0.54)$ levels [11], which had been based on outcomes from both upper and lower limb interventions combined.

The current meta-analysis of RCTs (published up to June 2017) showed strong evidence of meaningful change across the Body Structure/Function and Activity levels of the ICF-WHO, unlike earlier reviews $[46,58]$. First, we showed significant effects at the Body Structure/Function level, where the earlier review of Laver and colleagues showed no change on a group of "other outcomes" that were largely at this ICF-WHO level. Second, our review showed that the largest effect sizes were consistently identified at the Activity level whereas Palma and colleagues [58] found inconclusive support and Laver et al. [46] reported relatively small effects on upper-limb function $(d=0.28)$. Finally, treatment effects at the Participation level were small $(g=0.38)$ and non-significant. Variation in the magnitude of effect across studies in our review ( $g$ ranging from -0.37 to 2.04 over five studies) may reflect issues in the assessment of participation outcomes, which is currently an imprecise science [105].

\section{Cognitive outcomes}

While cognitive impairment is common post-stroke [16, 20, 27], and cognitive and motor systems overlap at a structural and functional level $[9,20]$, only four [53, 54, 91, 97] studies included in the current meta-analysis measured cognitive outcomes. While preliminary, the overall effect of VR on cognition was encouraging, with a mean effect size of $g=0.45$. The limited number of studies did not permit any conclusions about the superiority of either VE or CG approaches. Palma and colleagues [58] also reviewed cognitive outcomes (from four RCTs), but found no advantage for a VR approach. However, the relevance of several included studies was questionable. One study compared VR with a computerized cognitive rehabilitation program, not with physical therapy [106], a second study contained no identifiable cognitive outcome measures [107] and in a third study, the mental function under investigation was mood state, rather than cognitive status [75]. The fourth study was also included in the current meta-analysis [91]. The results of the current review appear more valid, and provide encouragement that VR can contribute to cognitive rehabilitation. Moving forward, researchers and clinicians are encouraged to be mindful of the inter-relationship between motor and cognitive systems [9] and the potential cognitive benefits of motor-based stroke rehabilitation using VR [25, 31, 54]. For example, a within-group study by Kizony et al. [51] found preliminary evidence supporting the interaction between motor and 
cognitive function in stroke patients undergoing VR. A more recent study by Subramanian and colleagues [50] provided further evidence of the association between cognitive and motor recovery. Moreover, it was shown that patient's psychological well-being can also affect motor learning using VR [50], and should also be taken into account in future studies of VR in stroke.

\section{Implementation parameters and design factors}

Dose-effect relationships remain inconclusive in the VR area, and in need of further investigation. Reviewing the literature published between 1999 and 2004, Crosbie and colleagues [60] found VR was most commonly delivered three times per week for 1$1.5 \mathrm{~h}$, over a 2-4 week period (i.e. 6-18 h total). Similarly, in their review of the literature from 2008 to 2015, Palma et al. [58] reported the average dose of VR was $17.6 \mathrm{~h}$ for upper limb motor function rehabilitation, and $13.2 \mathrm{~h}$ for motor activity rehabilitation. These trends were continued in the current review, with the average VR intervention comprised of $40 \mathrm{~min}$ sessions delivered three days per week for 6 weeks, for a total of approximately 12 h. However, there was large variability in these implementation parameters, with protocols providing up to $60 \mathrm{~min}$ sessions, up to five times per week, for as many as 36 sessions. While a higher number of repetitions and longer training times are argued to be more beneficial for motor learning [108], VR outcomes are argued not to be exclusively dependent on dose [46]. In the current review, moderator analysis also found no clear added benefit of higher doses or massed practice of VR, suggesting a ceiling after which gains plateau. While the dose of rehabilitation may not be the most important factor affecting recovery [109], the average intensity, frequency and duration of VR training identified in the current review appeared to provide an effective schedule for cognitive and motor function outcomes, while reducing the chance of participant fatigue or burn out that may occur under higher intensity training.

\section{Active versus passive control groups}

There was no difference in effect sizes $(g=0.45$ c.f. 0.48$)$ for interventions that utilized an active control group (i.e. additional conventional therapy beyond just treatment as usual) or a passive one (i.e. treatment as usual only). This was an unexpected finding as active control group designs are preferred for their capability to presumably control for Hawthorne effects and other biases when comparison groups are not balanced in terms of time in therapy. However, the current findings suggest that the use of a passive control group does not inflate the effect size for the intervention group. It also suggests that those treatment strategies embedded in active conditions may not add substantially to the training effects usually observed for treatment as usual. This finding provides credence to studies that lack the resourcing to implement an active control group design and just proceed with a treatment as usual group, which is the case more often than not in rehabilitation research [110].

\section{Sub-acute versus chronic stage}

Moderator analysis showed that VR administered in the sub-acute $(g=0.25)$ and the chronic stages $(g=$ 0.51 ) were both effective. However, only seven studies included in the current review intervened early after stroke, and the optimal time window for delivering VR remains an issue for further study. For the chronic group, there was a large variation in the time since stroke (range 6 months to several years). While it may be argued that participants with longer-term impairment remained responsive to VR treatment, early intervention is still recommended $[111,112]$ to address neurological changes before chronic disability ensues [101]. As particular treatment modalities are refined with advances in the technology (e.g., delivery of augmented feedback) there will be unique opportunities to enhance neuroplastic changes during this critical time period [113].

\section{Outcomes at follow-up}

A third of all studies included follow-up assessment [44, 74, 76, 79, 86-88, 90, 93-96]. Participant retention was generally high, with only one study experiencing attrition rates over $10 \%$ at follow-up [86]. Follow-up duration was four weeks in five studies $[44,76,79,86,94]$, six weeks in one study [96], eight weeks in two studies [90, 93], 13 weeks in three studies [74, 88, 95] and 26 weeks in one study [87]. Over all follow-up durations, the initial gains reported immediately following VR training were preserved. These findings are encouraging, and suggest that a discrete period of VR can affect longerlasting improvements in overall motor function, and on ICF-WHO Body Structure/Function and Activity level outcomes in particular. By comparison, there is accumulating evidence that early improvements after conventional rehabilitation may not be sustained long-term after stroke $[114,115]$. Notably, the current review showed that gains were maintained regardless of VR approach (CG or VE), dosing (i.e. frequency, intensity, or duration of training), or stage of recovery (i.e. sub-acute or chronic). Surprisingly, no studies examined cognitive outcomes at follow- 
up, and the durability of post-training improvements in this domain remains unknown. The stability of gains over periods longer than six months has also not been explored but should be encouraged in future research. Also for further study are questions about whether booster sessions or other strategies such as activity monitoring, goal setting, or feedback systems [116] are needed to optimize stroke survivors' longer-term outcomes after VR.

\section{Risk of bias}

To maximize the quality of evidence in this review, all of the included studies were Level 1b (RCTs) to Level 2b (small RCTs) according to the Centre for Evidence-Based Medicine [117]. As evaluated formally using the PEDro Scale, the quality of studies was also generally high. Not surprisingly, the only design component consistently omitted was the blinding of participants, which is difficult to achieve using novel and distinct interventions like VR [46]. One study described their methodology as a doubleblind procedure [82], but while participants may have been naïve to the intended outcomes of the study it is unlikely they were unaware of their group assignment (VR vs. passive control group). The current study did not include a search and review of unpublished (grey) literature, which could be important to account for publication bias (or file drawer effect) [118]. The current review specifically focused on published, peer-review articles to ensure the high quality of included data, but performed a fail-safe $N$ [119] calculation to account for missing studies and grey literature. Fail-safe $N$ value of 439: that is, 13 missing studies for every observed study would be required for the overall effect of VR to be nullified, further supporting the observed efficacy of VR. With the risk of biases minimized, we are confident that $\mathrm{VR}$, and in particular VE, can be recommended as a useful adjunct or alternative to conventional therapy when retraining motor and cognitive function following stroke. The ability of VR to enhance experiencedependent neuroplasticity is suggested but demands new research to investigate changes at the brain level. These recommendations are discussed below.

\section{Limitations and directions for future research}

The current review did not extend to a formal investigation of active ingredients (i.e. those aspects of VR that are having the most profound impact on functioning), which remains an important and unresolved issue in VR. What makes this issue particularly hard to dissect is the sheer variety in types of interface, augmented feedback, setting, and so on across different studies. It is likely there are both generic and more specific effects of VR on neuroplastic changes and the process of skill learning itself. For example, novelty and engagement are critical to any rehabilitation paradigm and can be captured by a number of well-designed (game-like) VE platforms, or popular CG systems like Wii, Kinect, and PlayStation [90]. The capability of VR to scale levels of difficulty and to provide appropriate rewards to users in the context of gameplay and advancement between levels is critical to CG. Use of augmented feedback (known to be important in motor learning) is one factor that will vary greatly with interface design and the type of humancomputer interaction that a given system affords [36]. Componential approaches to system evaluation will be particularly valuable in future research, varying a critical ingredient that is thought to predict an outcome while holding all other factors constant.

The effect of different neurological characteristics on VR rehabilitation outcomes is also in need of examination. Most studies in the current review used mixed samples of hemorrhagic and ischemic clients; only three studies sampled exclusively ischemic stroke patients [78, 86, 93]. Some studies suggest that hemorrhagic stroke may result in more severe cognitive, motor and functional impairment than ischemic stroke [120, 121]. By comparison, other work shows that differences between stroke types are marginal across these domains [122-124]. Future investigations would benefit from comparison of these stroke types to test the impact of mixed cohorts. Moreover, consistent reporting of details including lesion location (e.g. Oxfordshire Community Stroke Project Classification) and hemisphere, and initial severity and symptom profiles (e.g. National Institutes of Health Stroke Scale; modified Rankin Scale), will assist in identifying the neurological characteristics of stroke more or less responsive to VR.

Many studies in the current review had small participant numbers. With only an average of 15 participants per group, a number of studies lacked sufficient statistical power to examine more than one or two outcomes [125], and were likely underpowered to examine interactions, predictors, or multivariate effects. As we recommend examining outcomes across all three levels of the ICF-WHO, including cognitive outcomes, larger- $n$ studies are recommended in the future, with power calculations pointing to in excess of 20 participants per treatment arm.

Variation in the choice of primary outcome measure also limits comparison between studies. The VR research field should consider developing a consensus 
statement on evaluation research to aid the consistency in measurement. For example, at the Body Structure/Function level, the FMA might be considered as a "gold standard" in the absence of a better tool at this point in time. At the Activity level, the Box and Blocks Test has been shown to correlate very highly with longer test batteries that assess skill (like the Action Research Arm Test), and could be included as a standard, easy to administer measure. Due to a limited number of studies reporting cognitive outcomes, the current review could only report on cognition as a unified concept, rather than its more specific domains. Taken together, there is a need to include wellvalidated assessment of cognition.

The far transfer of training effects to important aspects of daily functioning, independence, and quality of life was examined in only five of the current studies, all of which utilized a VE approach [13, 44, 57, 87, 90]. The overall effect size on Participation outcomes was non-significant $(p=0.26)$. This result mirrored an earlier review by Saposnik and Levin [19] which identified only one study reporting on (social) participation. By comparison, the review of Lohse and colleagues [11] identified a single study that reported a significant effect on participation outcomes. However, Lohse and colleagues [11] misclassified the Jebsen Taylor Hand Function Test as a Participation measure when in fact it is usually classified as an Activity level outcome $[59,126]$. In our review we observed high variability in results, ranging from nonsignificance $(g=-0.37)$ to a large significant effect $(g$ $=2.04)$. The latter study was the only home-based intervention and involved a control group that completed their conventional rehabilitation before the study started. One or both of these unique features may explain the size of the observed effect. Overall, efficacy of VR at the Participation level of the ICFWHO remains inconclusive (see also [58]) and the amount of evidence bearing on it is very limited [19, 58]. We recommend examination of far-transfer Participation outcomes as standard practice.

\section{Implications for practice}

Knowledge of the pattern of treatment effects across ICF levels has important implications for the design of tailored interventions for stroke and evidencebased recommendations for care. Stronger effects for VE-based systems over CG suggest that the added expense of acquiring purpose-designed systems might be a good investment for clinicians, backed, of course, by well-controlled evaluation studies. However, at this point, we still do not have sufficient data to make strong predictions about the (far-transfer) effects of such training on Participation. In cases where cost and access to VE systems is an issue, CG systems will still leverage outcomes at the Body Structure/Function and Activity levels.

There is too little data to yet make firm conclusions about the impact of VR on cognition. However, there are a number of examples where cognitive performance has been enhanced through what are essentially motor-based interventions for the upper limbs. For example, for patients with traumatic brain injury (TBI), Mumford and colleagues [38] showed VR produces a significant subjective improvement in attention and memory function.

Taken together, clinicians and researchers alike are encouraged to seek out purpose-designed VE systems that can boast high-quality evidence for their efficacy. The principled and evidence-based approach to the design, implementation, and evaluation of VE instruments confers a considerable therapeutic advantage at the level of functional movement skill. As a matter of course, future research needs to extend the evaluation of outcomes across all ICF-WHO levels.

As mentioned, the moderator analysis was unable to detect a linear dose-response relationship, as no advantage for higher dosing on any of the VR approaches or rehabilitation outcomes were found. Future studies should seek to explore the more active ingredients of VR, to maximize both the efficacy and the efficiency of treatment rather than simply relying on higher doses. Implications for patient engagement, retention, and satisfaction remain to be explored.

\section{Conclusion}

The physical and cognitive impairment resulting from stroke is persistent and prominent, and the prospect of recovery both compelling and elusive. VR interventions offer the unique opportunity for patients to interact in an enriched environment, providing structured, scalable training opportunities augmented by multi-sensory feedback to enhance skill learning and neuroplasticity through repeated practice. Findings from this review suggest VR has an added advantage over conventional interventions, and can produce immediate and longer-term improvements in motor function and the performance of cognitive and motor activities following stroke. The evidence-based efficacy of a VR approach extends to patients in both the acute and chronic recovery stage, utilizing a spaced training schedule delivered via either purpose-designed or commercially available systems. Continued application of this promising technology is encouraged, to refine our understanding of the factors contributing to the beneficial effects of VR, and to promote the transfer of gains to participation outcomes. 


\section{Appendix}

Table 6 Sample search strategy for the Ovid MEDLINE database

\begin{tabular}{|c|c|}
\hline Set \# & Search String \\
\hline 1 & Cerebral Ischemia/ or exp. Cerebrovascular Accidents/ or exp Ischemia/ or exp Cardiovascular Disorders/ or Stroke.mp. \\
\hline 2 & $\begin{array}{l}\text { Cerebrovascular disease/ or exp basal ganglion hemorrhage/ or exp brain hematoma/ or exp brain hemorrhage/ or exp brain infarction/ } \\
\text { or exp brain ischemia/ or exp carotid artery disease/ or cerebral artery disease/ or exp cerebrovascular malformation/ or exp intracranial } \\
\text { aneurysm/ or exp occlusive cerebrovascular disease/ or stroke/ or stroke unit/ or stroke patient/ }\end{array}$ \\
\hline 3 & (Hemorrhag* or Subarachnoid Hemorrhage or intracranial hemorrhage).mp. \\
\hline 4 & (Poststroke or post-stroke or cerebrovasc* or cerebral vascular or brain vasc* or cerebral vasc* or cva* or apoplexy* or SAH).mp. \\
\hline 5 & (Brain* or cerebr* or cerebell* or intracran* or intracerebral N5 isch?emi* or infarct* or thrombo* or emboli* or occlus*).mp. \\
\hline 6 & 1 or 2 or 3 or 4 or 5 or 6 \\
\hline 7 & (Virtual real* or virtual-real* or VR).mp. \\
\hline 8 & (Augment* reality or virtual reality or augment* gam* or virtual gam*).mp. \\
\hline 9 & $\begin{array}{l}\text { (Video gam* or computer gam* or gaming consol* or interactive gam* or Nintendo Wii or kinect or nintendo or playstation or xbox or } \\
\text { gam* program).mp. }\end{array}$ \\
\hline 10 & (Virtual adj3 (environment* or object* or world* or treatment* or system* or program* or rehabilitation* or therap*)).mp. \\
\hline 11 & 8 or 9 or 10 or 11 \\
\hline 12 & 6 and 11 \\
\hline
\end{tabular}

\section{Additional files}

Additional file 1: Figure S1. Forest plot showing the overall main effect-sizes of Virtual rehabilitation after stroke for each individual study using the random-effects model. (TIFF $1240 \mathrm{~kb}$ )

Additional file 2: Figure S2. Forest plot showing the overall main effect-sizes for each individual study of virtual rehabilitation on the International Classification of Functioning and cognitive outcomes after stroke using the random-effects model. (TIFF $760 \mathrm{~kb}$ )

\section{Abbreviations}

ADLs: Activities of Daily Living; BBT: Box and Blocks Test; CG: Computerized Gaming; CT: Conventional Treatment; FMA: Fugl-Meyer Assessment; ICFWHO: The World Health Orgainzation's International Classification of Functioning, Disability, and Health; PEDro: Physiotherapy Evidence Database; RCT: Randomized Controlled Trial; VE: Virtual Environments; VR: Virtual Rehabilitation

\section{Acknowledgements}

Not applicable.

\section{Funding}

This research is supported by a Prince of Wales Hospital Foundation grant awarded to JR, and an Research Training Program award awarded to AA.

\section{Availability of data and materials}

Data and materials can be made available upon request to the authors.

\section{Authors' contributions}

All authors contributed to review and revision of the manuscript. JMR, PHW supervised the study. AA, JMR, PHW conceptualized and designed the study. JMR acquired the funding. AA acquired, processed, analyzed and interpreted the data from the study. AA, JMR, PHW drafted the original manuscript. AA, JMR, PHW, SM, KC finalized the manuscript. All authors read and approved the final manuscript.

Ethics approval and consent to participate Not applicable.
Consent for publication

Not applicable.

\section{Competing interests}

The authors report no competing interests.

\section{Publisher's Note}

Springer Nature remains neutral with regard to jurisdictional claims in published maps and institutional affiliations.

\section{Author details}

${ }^{1}$ School of Psychology, Faculty of Health Sciences, Australian Catholic University, Sydney, NSW, Australia. ${ }^{2}$ South Eastern Sydney Local Health District, Sydney, NSW, Australia. ${ }^{3}$ School of Psychology, Australian Catholic University, Melbourne, VIC, Australia. ${ }^{4}$ Centre for Disability and Development Research (CeDDR), Australian Catholic University, Melbourne, VIC, Australia.

Received: 24 September 2017 Accepted: 11 March 2018

Published online: 27 March 2018

\section{References}

1. World Health Organization: Global status report on noncommunicable diseases 2014

2. Feigin $\mathrm{VL}$, Forouzanfar $\mathrm{MH}$, Krishnamurthi $\mathrm{R}$, Mensah GA, Connor M, Bennett DA, Moran AE, Sacco RL, Anderson L, Truelsen T. Global and regional burden of stroke during 1990-2010: findings from the global burden of disease study 2010. Lancet. 2014;383:245-55.

3. Patel MD, Tilling K, Lawrence E, Rudd AG, Wolfe CDA, McKevitt C Relationships between long-term stroke disability, handicap and healthrelated quality of life. Age Ageing. 2006;35:273-9.

4. Mercierand C, Bourbonnais D. Relative shoulder fexor and handgrip strength is related to upper limb function after stroke. Clin Rehabil. 2004;18:215-21.

5. Hocine N, Gouaïch A, Cerri SA, Mottet D, Froger J, Laffont I. Adaptation in serious games for upper-limb rehabilitation: an approach to improve training outcomes. User Model User-adapt Interact. 2015;25:65-98.

6. Douiri A, Rudd AG, Wolfe CD. Prevalence of poststroke cognitive impairment. Stroke. 2013;44:138-45. 
7. Cumming T, Churilov L, Lindén T, Bernhardt J. Montreal cognitive assessment and mini-mental state examination are both valid cognitive tools in stroke. Acta Neurol Scand. 2013;128:122-9.

8. Cumming TB, Marshall RS, Lazar RM. Stroke, cognitive deficits, and rehabilitation: still an incomplete picture. Int J Stroke. 2013;8:38-45.

9. Kalaria RN, Akinyemi R, Ihara M. Stroke injury, cognitive impairment and vascular dementia. Biochim Biophys Acta. 2016;1862:915-25.

10. Lincoln N, Majid M, Weyman N. Cognitive rehabilitation for attention deficits following stroke. Cochrane Database Syst Rev. 2000;(4):CD002842.

11. Lohse KR, Hilderman CG, Cheung KL, Tatla S, Van der Loos HM. Virtual reality therapy for adults post-stroke: a systematic review and meta-analysis exploring virtual environments and commercial games in therapy. PLoS One. 2014;9:e93318.

12. Lawrence ES, Coshall C, Dundas R, Stewart J, Rudd AG, Howard R, Wolfe CD. Estimates of the prevalence of acute stroke impairments and disability in a multiethnic population. Stroke. 2001;32:1279-84.

13. Duff M, Chen Y, Attygalle S, Herman J, Sundaram H, Qian G, He J, Rikakis T. An adaptive mixed reality training system for stroke rehabilitation. IEEE Trans Neural Syst Rehabil Eng. 2010;18:531-41.

14. Leśniak M, Bak T, Czepiel W, Seniów J, Członkowska A. Frequency and prognostic value of cognitive disorders in stroke patients. Dement Geriatr Cogn Dis Extra. 2008:26:356-63.

15. Patel M, Coshall C, Rudd AG, Wolfe CD. Natural history of cognitive impairment after stroke and factors associated with its recovery. Clin Rehabil. 2003;17:158-66.

16. Wagle J, Farner L, Flekkøy K, Wyller TB, Sandvik L, Fure B, Stensrød B, Engedal K. Early post-stroke cognition in stroke rehabilitation patients predicts functional outcome at 13 months. Dement Geriatr Cogn Dis. 2011;31:379-87.

17. Rizzo AA, Schultheis M, Kerns KA, Mateer C. Analysis of assets for virtual reality applications in neuropsychology. Neuropsychol Rehabil. 2004;14:207-39.

18. Rose FD, Brooks BM, Rizzo AA. Virtual reality in brain damage rehabilitation: review. Cyberpsychol Behav. 2005;8:241-62.

19. Saposnik G, Levin M, Group SORCW. Virtual reality in stroke rehabilitation: a meta-analysis and implications for clinicians. Stroke. 2011:42:1380-6.

20. Zinn S, Dudley TK, Bosworth HB, Hoenig HM, Duncan PW, Horner RD. The effect of poststroke cognitive impairment on rehabilitation process and functional outcome. Arch Phys Med Rehabil. 2004;85:1084-90.

21. da Silva Cameirão M, Bermúdez i, Badia S, Duarte E, Verschure PF. Virtual reality based rehabilitation speeds up functional recovery of the upper extremities after stroke: a randomized controlled pilot study in the acute phase of stroke using the rehabilitation gaming system. Restor Neurol Neurosci. 2011;29:287-98.

22. Weiss PLT, Keshner EA, Levin MF. Virtual reality for physical and motor rehabilitation. New York: Springer; 2014.

23. Kim GJ. A SWOT analysis of the field of virtual reality rehabilitation and therapy. Presence Teleop Virt. 2005;14:119-46.

24. Al-Khindi T, Macdonald RL, Schweizer TA. Cognitive and functional outcome after aneurysmal subarachnoid hemorrhage. Stroke. 2010; 41(8):e519-36.

25. Weiss PL, Kizony R, Feintuch U, Katz N. Virtual reality in neurorehabilitation. Neurorehabil Neural Repair. 2006;51:182-97.

26. Clare L, Woods RT, Moniz Cook ED, Orrell M, Spector A. Cognitive rehabilitation and cognitive training for early-stage Alzheimer's disease and vascular dementia. Cochrane Database Syst Rev. 2003:4:CD003260.

27. Gates NJ, Sachdev PS, Singh MAF, Valenzuela M. Cognitive and memory training in adults at risk of dementia: a systematic review. BMC Geriatr. 2011;11:55.

28. Mumford N, Wilson PH. Virtual reality in acquired brain injury upper limb rehabilitation: evidence-based evaluation of clinical research. Brain Inj. 2009;23:179-91.

29. Taylor MJ, McCormick D, Shawis T, Impson R, Griffin M. Activitypromoting gaming systems in exercise and rehabilitation. J Rehabil Res Dev. 2011;48:1171-86.

30. Fritz SL, Peters DM, Merlo AM, Donley J. Active video-gaming effects on balance and mobility in individuals with chronic stroke: a randomized controlled trial. Top Stroke Rehabil. 2013:20:218-25.

31. Kizony R, Katz N. Adapting an immersive virtual reality system for rehabilitation. Comput Animat Virtual Worlds. 2003;14:261-8.

32. Edmans JA, Gladman JR, Cobb S, Sunderland A, Pridmore T, Hilton D, Walker MF. Validity of a virtual environment for stroke rehabilitation. Stroke. 2006;37:2770-5.

33. Levin MF. Can virtual reality offer enriched environments for rehabilitation? Expert Rev Neurother. 2011;11:153-5.
34. Claessen MH, van der Ham IJ, Jagersma E, Visser-Meily JM. Navigation strategy training using virtual reality in six chronic stroke patients: a novel and explorative approach to the rehabilitation of navigation impairment. Neuropsychol Rehabil. 2016;26:822-46.

35. Edmans J, Gladman J, Hilton D, Walker M, Sunderland A, Cobb S, Pridmore T, Thomas S. Clinical evaluation of a non-immersive virtual environment in stroke rehabilitation. Clin Rehabil. 2009;23:106-16.

36. Duncan F, Kutlubaev MA, Dennis MS, Greig C, Mead GE. Fatigue after stroke: a systematic review of associations with impaired physical fitness. Int J Stroke. 2012;7:157-62.

37. Schepers VP, Visser-Meily AM, Ketelaar M, Lindeman E. Poststroke fatigue: course and its relation to personal and stroke-related factors. Arch Phys Med Rehabil. 2006:87:184-8.

38. Mumford N, Duckworth J, Thomas PR, Shum D, Williams G, Wilson PH. Upper-limb virtual rehabilitation for traumatic brain injury: a preliminary within-group evaluation of the elements system. Brain Inj. 2012;26:166-76.

39. Wilson P, Green D, Caeyenberghs K, Steenbergen B, Duckworth J. Integrating new technologies into the treatment of CP and DCD. Curr Dev Disord Rep. 2016:3:138-51.

40. Duckworth J, Mumford N, Caeyenberghs K, Eldridge R, Mayson S, Thomas PR, Shum D, Williams G, Wilson PH. Resonance: an interactive tabletop artwork for co-located group rehabilitation and play. In: International conference on universal access in human-computer interaction. Cham: Springer; 2015. p. 420-31.

41. Duckworth J, Thomas PR, Shum D, Wilson PH. Designing co-located tabletop interaction for rehabilitation of brain injury. In: International conference of design, user experience, and usability. Berlin: Springer; 2013. p. 391-400.

42. Subramanian SK, Lourenço CB, Chilingaryan G, Sveistrup H, Levin MF. Arm motor recovery using a virtual reality intervention in chronic stroke: randomized control trial. Neurorehabil Neural Repair. 2013;27:13-23.

43. Subramanian S, Knaut LA, Beaudoin C, McFadyen BJ, Feldman AG, Levin MF. Virtual reality environments for post-stroke arm rehabilitation. Neuroeng Rehabil. 2007:4:20

44. Yin CW, Sien NY, Ying LA, Chung SF-CM, Tan May Leng D. Virtual reality for upper extremity rehabilitation in early stroke: a pilot randomized controlled trial. Clin Rehabil. 2014:28:1107-14.

45. Green D, Wilson PH. Use of virtual reality in rehabilitation of movement in children with hemiplegia- a multiple case study evaluation. Disabil Rehabil. 2012;34:593-604.

46. Laver KE, George S, Thomas S, Deutsch JE, Crotty M. Virtual reality for stroke rehabilitation. Cochrane Database Syst Rev. 2015;(2):CD008349.

47. Cicerone KD, Langenbahn DM, Braden C, Malec JF, Kalmar K, Fraas M, Felicetti T, Laatsch L, Harley JP, Bergquist T. Evidence-based cognitive rehabilitation: updated review of the literature from 2003 through 2008. Arch Phys Med Rehabil. 2011;92:519-30.

48. Nys G, Van Zandvoort M, De Kort P, Jansen B, De Haan E, Kappelle L. Cognitive disorders in acute stroke: prevalence and clinical determinants. Cerebrovasc Dis. 2007;23:408-16.

49. Chen C, Leys D, Esquenazi A. The interaction between neuropsychological and motor deficits in patients after stroke. Neurology. 2013;80:527-34.

50. Subramanian SK, Chilingaryan G, Levin MF, Sveistrup H. Influence of training environment and cognitive deficits on use of feedback for motor learning in chronic stroke. In: Virtual Rehabilitation Proceedings (ICVR). New York: 2015 International Conference on: IEEE; 2015. p. 38-43.

51. Kizony R, Katz N, Weiss P. Virtual reality based intervention in rehabilitation: relationship between motor and cognitive abilities and performance within virtual environments for patients with stroke. In: Proceedings of the 5th international conference on disability, virtual reality and associated technology Oxford, UK; 2004

52. Mullick AA, Subramanian SK, Levin MF. Emerging evidence of the association between cognitive deficits and arm motor recovery after stroke: a meta-analysis. Restor Neurol Neurosci. 2015;33:389-403.

53. Kim BR, Chun MH, Kim LS, Park JY. Effect of virtual reality on cognition in stroke patients. Ann Rehabil Med. 2011;35:450-9.

54. Gamito P, Oliveira J, Coelho C, Morais D, Lopes P, Pacheco J, Brito R, Soares F, Santos N, Barata AF. Cognitive training on stroke patients via virtual reality-based serious games. Disabil Rehabil. 2015;39:385-8.

55. World Health Organization. International classification of functioning, disability and health: ICF. Geneva: World health Organization; 2017.

56. Vargus-Adams JN, Majnemer A. International classification of functioning, disability and health (ICF) as a framework for change: revolutionizing rehabilitation. J Child Neurol. 2014;29:1030-5. 
57. Levin MF, Snir O, Liebermann DG, Weingarden H, Weiss PL. Virtual reality versus conventional treatment of reaching ability in chronic stroke: clinical feasibility study. Neurol Ther. 2012;1:3.

58. Palma GCS, Freitas TB, Bonuzzi GMG, Soares MAA, Leite PHW, Mazzini NA Almeida MRG, Pompeu JE, Torriani-Pasin C. Effects of virtual reality for stroke individuals based on the international classification of functioning and health: a systematic review. Top Stroke Rehabil. 2017;24:269-78.

59. Murphy MA, Resteghini C, Feys P, Lamers I. An overview of systematic reviews on upper extremity outcome measures after stroke. BMC Neurol. 2015:15:29.

60. Crosbie J, Lennon S, Basford J, McDonough S. Virtual reality in stroke rehabilitation: still more virtual than real. Disabil Rehabil. 2007;29:1139-46.

61. Henderson A, Korner-Bitensky N, Levin M. Virtual reality in stroke rehabilitation: a systematic review of its effectiveness for upper limb motor recovery. Top Stroke Rehabil. 2007;14:52-61.

62. Viñas-Diz S, Sobrido-Prieto M. Virtual reality for therapeutic purposes in stroke: a systematic review. Neurología (English Edition). 2016;31:255-77.

63. Cavalcanti Moreira M, de Amorim Lima AM, Ferraz KM, Benedetti Rodrigues MA. Use of virtual reality in gait recovery among post stroke patients-a systematic literature review. Disabil Rehabil Assist Technol. 2013;8:357-62.

64. Mundy L, Hiller J. Rehabilitation of stroke patients using virtual reality games. Australia and New Zealand Horizon Scanning Network Prioritising Summary. Canberra: Commonwealth of Australia; 2010. p. 27.

65. Laver KE, Lange B, George S, Deutsch JE, Saposnik G, Crotty M. Virtual reality for stroke rehabilitation. Cochrane Database Syst Rev. 2017;(11):CD008349. https://doi.org/10.1002/14651858.

66. Wahl A-S, Schwab ME. Finding an optimal rehabilitation paradigm after stroke: enhancing fiber growth and training of the brain at the right moment. Front Hum Neurosci. 2014;8:381. https://doi.org/10.3389/fnhum.2014.00381.

67. Liberati A, Altman DG, Tetzlaff J, Mulrow C, Gøtzsche PC, loannidis JP, Clarke M, Devereaux PJ, Kleijnen J, Moher D. The PRISMA statement for reporting systematic reviews and meta-analyses of studies that evaluate health care interventions: explanation and elaboration. PLoS Med. 2009;6:e1000100.

68. Maher CG, Sherrington C, Herbert RD, Moseley AM, Elkins M. Reliability of the PEDro scale for rating quality of randomized controlled trials. PTJ. 2003; 83:713-21.

69. Kamper SJ, Moseley AM, Herbert RD, Maher CG, Elkins MR, Sherrington C. 15 years of tracking physiotherapy evidence on PEDro, where are we now? 2015

70. Sherrington C, Moseley AM, Herbert RD, Maher CG. Evidence for physiotherapy practice: a survey of the physiotherapy evidence database (PEDro). Aust J Physiother. 2002;48:43-9.

71. Cohen J. Statistical power analysis for the behavioral sciences. 2nd ed. Hilldale: 1988 Erlbaum; 1988.

72. Rosenthal R. Writing meta-analytic reviews. Psychol Bull. 1995;118:183.

73. da Silva Ribeiro NM, Ferraz DD, Pedreira É, Pinheiro Í, da Silva Pinto AC, Neto MG, dos Santos LRA, Pozzato MGG, Pinho RS, Masruha MR. Virtual rehabilitation via Nintendo $\mathrm{Wii}^{\circledast}$ and conventional physical therapy effectively treat post-stroke hemiparetic patients. Top Stroke Rehabil. 2015;22:299-305.

74. Kong K-H, Loh Y-J, Thia E, Chai A, Ng C-Y, Soh Y-M, Toh S, Tjan S-Y. Efficacy of a virtual reality commercial gaming device in upper limb recovery after stroke: a randomized, controlled study. Top Stroke Rehabil. 2016;23:333-40

75. Shin J-H, Park SB, Jang SH. Effects of game-based virtual reality on healthrelated quality of life in chronic stroke patients: a randomized, controlled study. Comput Biol Med. 2015;63:92-8.

76. Shin J-H, Ryu H, Jang SH. A task-specific interactive game-based virtual reality rehabilitation system for patients with stroke: a usability test and two clinical experiments. J Neuroeng Rehabil. 2014;11:32.

77. Kiper P, Piron L, Turolla A, Stożek J, Tonin P. The effectiveness of reinforced feedback in virtual environment in the first 12 months after stroke. Neurol Neurochir Pol. 2011;45:436-44.

78. Piron L, Turolla A, Agostini M, Zucconi CS, Ventura L, Tonin P, Dam M. Motor learning principles for rehabilitation: a pilot randomized controlled study in poststroke patients. Neurorehabil Neural Repair. 2010;24:501-8.

79. Kiper P, Agostini M, Luque-Moreno C, Tonin P, Turolla A. Reinforced feedback in virtual environment for rehabilitation of upper extremity dysfunction after stroke: preliminary data from a randomized controlled trial. Biomed Res Int. 2014;752128. https://doi.org/10.1155/2014/752128.

80. Kim EK, Kang JH, Park JS, Jung BH. Clinical feasibility of interactive commercial Nintendo gaming for chronic stroke rehabilitation. J Phys Ther Sci. 2012;24:901-3.
81. Lee $\mathrm{G}$. Effects of training using video games on the muscle strength, muscle tone, and activities of daily living of chronic stroke patients. J Phys Ther Sci. 2013;25:595-7.

82. Kwon J-S, Park M-J, Yoon I-J, Park S-H. Effects of virtual reality on upper extremity function and activities of daily living performance in acute stroke: a double-blind randomized clinical trial. NeuroRehabilitation. 2012;31:379-85.

83. Chen M-H, Huang L-L, Lee C-F, Hsieh C-L, Lin Y-C, Liu H, Chen M-I, Lu W-S. A controlled pilot trial of two commercial video games for rehabilitation of arm function after stroke. Clin Rehabil. 2015;29:674-82.

84. Assis GA, Corrêa AG, Martins MB, Pedrozo WG, Lopes Rde D. An augmented reality system for upper-limb post-stroke motor rehabilitation: A feasibility study. Disabil Rehabil Assist Technol. 2016;11:521-8.

85. In TS, Jung KS, Lee SW, Song CH. Virtual reality reflection therapy improves motor recovery and motor function in the upper extremities of people with chronic stroke. J Phys Ther Sci. 2012;24:339-43.

86. Saposnik G, Cohen LG, Mamdani M, Pooyania S, Ploughman M, Cheung D, Shaw J, Hall J, Nord P, Dukelow S. Efficacy and safety of non-immersive virtual reality exercising in stroke rehabilitation (EVREST): a randomised, multicentre, single-blind, controlled trial. The Lancet Neurol. 2016;15:1019-27.

87. Housman SJ, Scott KM, Reinkensmeyer DJ. A randomized controlled trial of gravity-supported, computer-enhanced arm exercise for individuals with severe hemiparesis. Neurorehabil Neural Repair. 2009;23:505-14.

88. Yavuzer G, Senel A, Atay M, Stam H. Playstation eyetoy games improve upper extremity-related motor functioning in subacute stroke: a randomized controlled clinical trial. Eur J Phys Rehabil Med. 2008:44:237-44.

89. $\operatorname{Sin} \mathrm{H}$, Lee $\mathrm{G}$. Additional virtual reality training using Xbox Kinect in stroke survivors with hemiplegia. Am J Phys Med Rehabil. 2013;92:871-80.

90. Standen P, Threapleton K, Richardson A, Connell L, Brown D, Battersby S, Platts F, Burton A. A low cost virtual reality system for home based rehabilitation of the arm following stroke: a randomised controlled feasibility trial. Clin Rehabil. 2017;31:340-50.

91. Choi JH, Han EY, Kim BR, Kim SM, Im SH, Lee SY, Hyun CW. Effectiveness of commercial gaming-based virtual reality movement therapy on functional recovery of upper extremity in subacute stroke patients. Ann Rehabil Med. 2014;38:485-93.

92. Broeren J, Claesson L, Goude D, Rydmark M, Sunnerhagen KS. Virtual rehabilitation in an activity Centre for community-dwelling persons with stroke. Cerebrovasc Dis. 2008;26:289-96.

93. Piron L, Turolla A, Agostini M, Zucconi C, Cortese F, Zampolini M, Zannini M, Dam M, Ventura L, Battauz M. Exercises for paretic upper limb after stroke: a combined virtual-reality and telemedicine approach. J Rehabil Med. 2009;41:1016-20.

94. Kottink Al, Prange GB, Krabben T, Rietman JS, Buurke JH. Gaming and conventional exercises for improvement of arm function after stroke: a randomized controlled pilot study. Games Health J. 2014;3:184-91.

95. Givon N, Zeilig G, Weingarden H, Rand D. Video-games used in a group setting is feasible and effective to improve indicators of physical activity in individuals with chronic stroke: a randomized controlled trial. Clin Rehabil. 2016:30:383-92.

96. Crosbie J, Lennon S, McGoldrick M, McNeill M, McDonough S. Virtual reality in the rehabilitation of the arm after hemiplegic stroke: a randomized controlled pilot study. Clin Rehabil. 2012;26:798-806.

97. Kihoon J, Yu J, Jung J. Effects of virtual reality-based rehabilitation on upper extremity function and visual perception in stroke patients: a randomized control trial. J Phys Ther Sci. 2012;24:1205-8.

98. Rothstein HR, Sutton AJ, Borenstein M. Publication bias in meta-analysis: prevention, assessment and adjustments. Chichester: Wiley; 2006.

99. Egger M, Smith GD, Schneider M, Minder C. Bias in meta-analysis detected by a simple, graphical test. BMJ. 1997;315:629-34.

100. Langhorne $P$, Coupar F, Pollock A. Motor recovery after stroke: a systematic review. Lancet Neurol. 2009;8:741-54.

101. Pollock A, Baer G, Campbell P, Choo PL, Forster A, Morris J, Pomeroy VM, Langhorne $P$. Physical rehabilitation approaches for the recovery of function and mobility after stroke. Stroke. 2014;45:e202.

102. Teasell R, Meyer MJ, McClure A, Pan C, Murie-Fernandez M, Foley N, Salter K. Stroke rehabilitation: an international perspective. Top Stroke Rehabil. 2009;16:44-56.

103. Norouzi-Gheidari N, Archambault PS, Fung J. Effects of robot-assisted therapy on stroke rehabilitation in upper limbs: systematic review and meta-analysis of the literature. J Rehabil Res Dev. 2012;49:479.

104. Stucki G, Ewert T, Cieza A. Value and application of the ICF in rehabilitation medicine. Disabil Rehabil. 2002;25:628-34. 
105. Imms C, Granlund M, Wilson PH, Steenbergen B, Rosenbaum PL, Gordon AM. Participation, both a means and an end: a conceptual analysis of processes and outcomes in childhood disability. Dev Med Child Neurol. 2016:59(1):16-25.

106. Kang SH, Kim D-K, Seo KM, Choi KN, Yoo JY, Sung SY, Park HJ. A computerized visual perception rehabilitation programme with interactive computer interface using motion tracking technology: a randomized controlled, single-blinded, pilot clinical trial study. Clin Rehabil. 2009;23:434-44.

107. Lee SJ, Chun MH. Combination transcranial direct current stimulation and virtual reality therapy for upper extremity training in patients with subacute stroke. Arch Phys Med Rehabil. 2014;95:431-8.

108. Muratori LM, Lamberg EM, Quinn L, Duff SV. Applying principles of motor learning and control to upper extremity rehabilitation. J Hand Ther. 2013;26:94-103.

109. Winstein CJ, Wolf SL, Dromerick AW, Lane CJ, Nelsen MA, Lewthwaite R, Cen SY, Azen SP. Effect of a task-oriented rehabilitation program on upper extremity recovery following motor stroke: the ICARE randomized clinical trial. J Am Med Assoc. 2016;315:571-81.

110. Lampit A, Hallock H, Valenzuela M. Computerized cognitive training in cognitively healthy older adults: a systematic review and meta-analysis of effect modifiers. PLoS Med. 2014;11:e1001756.

111. Hakkennes SJ, Brock K, Hill KD. Selection for inpatient rehabilitation after acute stroke: a systematic review of the literature. Arch Phys Med Rehabil. 2011:92:2057-70

112. Musicco M, Emberti L, Nappi G, Caltagirone C, Patients IMSoOoRoN. Early and long-term outcome of rehabilitation in stroke patients: the role of patient characteristics, time of initiation, and duration of interventions. Arch Phys Med Rehabil. 2003;84:551-8.

113. Adams HP, Del Zoppo G, Alberts MJ, Bhatt DL, Brass L, Furlan A, Grubb RL, Higashida RT, Jauch EC, Kidwell C. Guidelines for the early management of adults with ischemic stroke. Circulation. 2007;115:e478-534.

114. Meyer S, Verheyden G, Brinkmann N, Dejaeger E, De Weerdt W, Feys H, Gantenbein AR, Jenni W, Laenen A, Lincoln N. Functional and motor outcome 5 years after stroke is equivalent to outcome at 2 months. Stroke. 2015:46:1613-9.

115. Löfgren B, Nyberg L, Mattsson M, Gustafson Y. Three years after in-patient stroke rehabilitation: a follow-up study. Cerebrovasc Dis. 1999;9:163-70.

116. Peek K, Sanson-Fisher R, Mackenzie L, Carey M. Interventions to aid patient adherence to physiotherapist prescribed self-management strategies: a systematic review. Physiotherapy. 2016;102:127-35.

117. Howick J, Chalmers I, Glasziou P, Greenhalgh T, Heneghan C, Liberati A, Moschetti I, Phillips B, Thornton H. Explanation of the 2011 Oxford Centre for Evidence-Based Medicine (OCEBM) levels of evidence Oxford center for Evidence-Based Medicine 2011

118. Rosenberg MS. The file-drawer problem revisited: a general weighted method for calculating fail-safe numbers in meta-analysis. Evolution. 2005: 59:464-8.

119. Rosenthal R. The file drawer problem and tolerance for null results. Psychol Bull. 1979;86:638.

120. Bhalla A, Wang Y, Rudd A, Wolfe CD. Differences in Outcome and Predictors between ischemic and intracerebral hemorrhage. Stroke. 2013;44:2174-81.

121. Andersen KK, Olsen TS, Dehlendorff C, Kammersgaard LP. Hemorrhagic and ischemic strokes compared. Stroke. 2009;40:2068-72.

122. Aminov A, Rogers JM, Johnstone SJ, Middleton S, Wilson PH. Acute single channel EEG predictors of cognitive function after stroke. PLoS One. 2017; 12:e0185841.

123. Crichton SL, Bray BD, McKevitt C, Rudd AG, Wolfe CD. Patient outcomes up to 15 years after stroke: Survival, disability, quality of life, cognition and mental health. J Neurol Neurosurg Psychiatry. 2016;87(10):1091-8. jnnp2016-313361

124. Cumming TB, Bernhardt J, Linden T. The Montreal cognitive assessment: short cognitive evaluation in a large stroke trial. Stroke. 2011;42:2642-4.

125. Tabachnick BG, Fidell LS, Osterlind SJ. Using multivariate. New York: Statistics; 2001.

126. Santisteban L, Térémetz M, Bleton J-P, Baron J-C, Maier MA, Lindberg PG Upper limb outcome measures used in stroke rehabilitation studies: a systematic literature review. PLoS One. 2016;11:e0154792.

\section{Submit your next manuscript to BioMed Central and we will help you at every step:}

- We accept pre-submission inquiries

- Our selector tool helps you to find the most relevant journal

- We provide round the clock customer support

- Convenient online submission

- Thorough peer review

- Inclusion in PubMed and all major indexing services

- Maximum visibility for your research

Submit your manuscript at www.biomedcentral.com/submit

) Biomed Central 